BNL-96853-2012-IR

$\mathrm{C}-\mathrm{A} / \mathrm{AP} / \# 448$

Jan. 2012

\title{
Simulations of RF capture with barrier bucket in booster at injections
}

\author{
C.J. Gardner
}

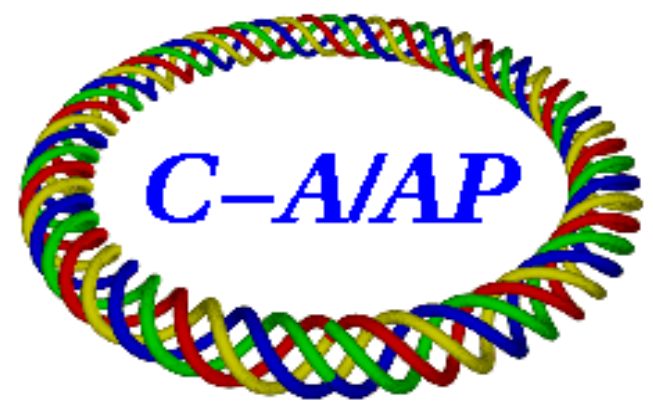

Collider-Accelerator Department Brookhaven National Laboratory

Upton, NY 11973

Notice: This document has been authorized by employees of Brookhaven Science Associates, LLC under Contract No. DE-AC02-98CH10886 with the U.S. Department of En ergy. The United States Government retains a nonexclusive, paid-up, irrevocable, world-wide license to publish or reproduce the published form of this document, or allow others to do so, for United States Government purposes. 


\title{
Simulations of RF Capture with Barrier Bucket in Booster at Injection
}

\author{
C.J. Gardner
}

January 23, 2012

As part of the effort to increase the number of ions per bunch in RHIC, a new scheme for RF capture of EBIS ions in Booster at injection has been developed. The scheme was proposed by M. Blaskiewicz and J.M. Brennan. It employs a barrier bucket to hold a half turn of beam in place during capture into two adjacent harmonic 4 buckets. After acceleration, this allows for 8 transfers of 2 bunches from Booster into 16 buckets on the AGS injection porch. During the Fall of 2011 the necessary hardware was developed and implemented by the RF and Controls groups. The scheme is presently being commissioned by K.L. Zeno with Au32+ ions from EBIS [1].

In this note we carry out simulations of the RF capture. These are meant to serve as benchmarks for what can be achieved in practice. They also allow for an estimate of the longitudinal emittance of the bunches on the AGS injection porch.

\section{Turn-by-Turn Equations of Motion}

Consider a ring in which particles are circulating with the guide field held constant. The synchronous particle has constant angular frequency $\omega_{s}$ and constant radius $R_{s}$. These give

$$
\beta_{s}=R_{s} \omega_{s} / c, \quad \gamma_{s}=1 / \sqrt{1-\beta_{s}^{2}}
$$

and

$$
\eta_{s}=\frac{1}{\gamma_{t}^{2}}-\frac{1}{\gamma_{s}^{2}}, \quad E_{s}=m c^{2} \gamma_{s}
$$


where $\gamma_{t}$ is the transition gamma of the ring, $m$ is the particle mass, and $c$ is the velocity of light. Let $t_{n}$ be the arrival time of a beam particle with respect to the synchronous particle on the $n$th turn around the ring. Let $E_{n}$ be the corresponding energy of the beam particle and define

$$
\phi_{n}=\omega_{s} t_{n}, \quad W_{n}=\frac{E_{n}-E_{s}}{\omega_{s}} .
$$

Then the turn-by-turn equations for the longitudinal motion of the beam particle are $[2,3]$

$$
\phi_{n+1}=\phi_{n}+a T_{s} W_{n}
$$

and

$$
W_{n+1}=W_{n}+T_{s} F\left(\phi_{n+1}\right)
$$

where

$$
a=\frac{\omega_{s}^{2} \eta_{s}}{\beta_{s}^{2} E_{s}}=\frac{c^{2} \eta_{s}}{R_{s}^{2} E_{s}}
$$

and

$$
T_{s}=\frac{2 \pi}{\omega_{s}} .
$$

The "force" function $F(\phi)$ depends on the excitation of RF cavities in the ring. Its explicit form is given in Section 3. For EBIS ions in Booster at injection we have

$$
T_{s}=10.410 \mu \mathrm{s}, \quad R_{s}=201.780 /(2 \pi) \mathrm{m}, \quad \gamma_{t}=4.832 .
$$

\section{Approximate Constant of Motion}

Consider the hamiltonian

$$
H(\phi, W)=\frac{1}{2} a W^{2}+U(\phi)
$$

where

$$
\frac{\partial U}{\partial \phi}=-F(\phi) .
$$

The equations of motion are

$$
\frac{d \phi}{d t}=\frac{\partial H}{\partial W}=a W
$$




$$
\frac{d W}{d t}=-\frac{\partial H}{\partial \phi}=F(\phi)
$$

and we have

$$
\frac{d H}{d t}=\frac{\partial H}{\partial t}=\frac{\partial U}{\partial t}
$$

The hamiltonian is therefore a constant of the motion if $U$ has no explicit dependence on time.

First-order symplectic integration [4] of (11) and (12) from time $t$ to time $t+T_{s}$ yields

$$
\phi\left(t+T_{s}\right)=\phi(t)+a T_{s} W(t)
$$

and

$$
W\left(t+T_{s}\right)=W(t)+T_{s} F\left(\phi\left(t+T_{s}\right)\right) .
$$

These are the same as (4) and (5). This suggests that the motion generated by the hamiltonian approximates that generated by (4) and (5). To the extent that this is true, the turn-by-turn quantity

$$
H\left(\phi_{n}, W_{n}\right)=\frac{1}{2} a W_{n}^{2}+U\left(\phi_{n}\right)
$$

obtained from (4) and (5) will be an approximate constant of the motion. We then expect that the regions of stable motion generated by (4) and (5) will be approximated by those associated with $H$. These regions are the RF buckets, which are calculated in Section 5.

\section{The "Force" Function $F(\phi)$}

The barrier bucket in Booster is produced by $\mathrm{RF}$ cavities in the A6 and E6 straight sections; the harmonic 4 buckets are produced by cavities in the A3 and B3 straights. These cavities are excited such that the function $F(\phi)$ has the form

$$
F(\phi)=\Delta(\phi) B \sin \chi-A \sin 4 \phi
$$

where

$$
B=\frac{e Q V_{B}}{2 \pi}, \quad A=\frac{e Q V_{A}}{2 \pi}
$$

and

$$
B \geq 0, \quad A \geq 0
$$


Here $e$ is the elementary charge, $e Q$ is the particle charge, and $V_{B}$ and $V_{A}$ are the amplitudes of the gap voltage per turn for the barrier and harmonic 4 buckets respectively. The function $\Delta(\phi)$ and phase $\chi$ are defined as follows.

1. For

$$
-\frac{\lambda}{2}-\frac{\Gamma}{2} \leq \phi \leq-\frac{\lambda}{2}+\frac{\Gamma}{2}
$$

we take

$$
\Delta(\phi)=1
$$

and

$$
\chi=\frac{2 \pi}{\Gamma}\left\{\phi+\frac{\lambda}{2}+\frac{\Gamma}{2}\right\}
$$

2. For

$$
\frac{\lambda}{2}-\frac{\Gamma}{2} \leq \phi \leq \frac{\lambda}{2}+\frac{\Gamma}{2}
$$

we take

$$
\Delta(\phi)=1
$$

and

$$
\chi=\frac{2 \pi}{\Gamma}\left\{\phi-\frac{\lambda}{2}+\frac{\Gamma}{2}\right\}
$$

3. Otherwise we take

$$
\Delta(\phi)=0
$$

Here $\Gamma$ is width of the barrier cavity pulses and $\lambda$ is the distance between the centers of the pulses.

Figure 1 shows a plot of the harmonic 4 excitation, $-A \sin 4 \phi$, and the barrier bucket excitation, $\Delta(\phi) B \sin \chi$, for the case in which

$$
A=0.2, \quad B=2.0, \quad \Gamma=0.2 \pi
$$

and

$$
\lambda=\pi+\Gamma .
$$

Note that the region of zero voltage between the two barrier pulses starts and ends where the harmonic 4 voltage crosses zero. This allows a half turn of beam to be held in place by the barrier bucket and be captured into two harmonic 4 buckets as the harmonic 4 voltage is raised from zero. Figure 2 shows the sum $F(\phi)=\Delta(\phi) B \sin \chi-A \sin 4 \phi$. 


\section{Fixed Points of the Motion}

To obtain the desired $F(\phi)$ we take the "potential" $U(\phi)$ to be

$$
U=\frac{A}{4}(1-\cos 4 \phi)+\Delta(\phi) \frac{B \Gamma}{2 \pi}(\cos \chi-1)
$$

which gives

$$
\frac{\partial U}{\partial \phi}=A \sin 4 \phi-\Delta(\phi) B \sin \chi
$$

and

$$
\frac{\partial^{2} U}{\partial \phi^{2}}=4 A \cos 4 \phi-\Delta(\phi) \frac{2 \pi}{\Gamma} B \cos \chi
$$

The constant terms in (29) have been chosen so that

$$
U(0)=0
$$

and so that $U(\phi)$ is continuous at

$$
\phi=-\frac{\lambda}{2}-\frac{\Gamma}{2}, \quad-\frac{\lambda}{2}+\frac{\Gamma}{2}, \quad \frac{\lambda}{2}-\frac{\Gamma}{2}, \quad \frac{\lambda}{2}+\frac{\Gamma}{2} .
$$

The fixed points of the motion are given by

$$
-\frac{\partial U}{\partial \phi}=0, \quad W=0 .
$$

Below transition $\left(\eta_{s}<0\right)$, the motion near a fixed point will be stable if

$$
-\frac{\partial^{2} U}{\partial \phi^{2}}>0
$$

and unstable if

$$
-\frac{\partial^{2} U}{\partial \phi^{2}}<0
$$

Figure 3 shows $-U(\phi)$ for the case

$$
A=0.2, \quad B=2.0, \quad \Gamma=0.2 \pi .
$$

Here the largest maxima of $-U$ occur at

$$
\phi= \pm 0.5970 \pi \text {. }
$$

These are unstable fixed points. The points

$$
\phi=0, \pm \pi / 2, \pm \pi
$$

are also unstable fixed points. The points

$$
\phi= \pm \pi / 4, \pm 3 \pi / 4
$$

are stable fixed points. 


\section{The RF Buckets}

For any unstable fixed point $\phi_{u}$, let $H_{u}$ be the value of $H(\phi, W)$ at

$$
\phi=\phi_{u}, \quad W=0
$$

Then

$$
H_{u}=U\left(\phi_{u}\right)
$$

and the equation

$$
H(\phi, W)=H_{u}
$$

defines separatrices $W(\phi)$. Solving (43) for $W^{2}(\phi)$ we obtain

$$
W^{2}(\phi)=\frac{2}{a}\left\{U\left(\phi_{u}\right)-U(\phi)\right\} .
$$

The outer brown curves in Figure $\mathbf{4}$ are the separatrices that pass through the unstable fixed points

$$
\phi_{u}= \pm 0.5970 \pi
$$

These curves form the barrier bucket. The inner brown curves are the separatrices that pass through the unstable fixed points

$$
\phi_{u}=0, \pm \pi / 2, \pm \pi
$$

These curves form the harmonic 4 buckets. Note that the barrier bucket is distorted by the harmonic 4 voltage, as are two of the harmonic 4 buckets by the barrier bucket voltage. Figure 5 shows $-U(\phi)$ and the barrier bucket for the case in which the harmonic 4 voltage is zero.

\section{Initial Particle Distribution}

The initial particle distribution for the simulation of RF capture is an 80-by- 80 array of points uniformly distributed over the region

$$
-\phi_{I} \leq \phi \leq \phi_{I}, \quad-W_{I} \leq W \leq W_{I}
$$

where

$$
\frac{\pi}{2} \leq \phi_{I} \leq \pi
$$


and

$$
W_{I}=\frac{E_{I}-E_{s}}{\omega_{s}}=\frac{\Delta E_{I}}{\omega_{s}} .
$$

Here $\Delta E_{I}$ is the energy half-width of the region. This gives fractional momentum half-width

$$
\frac{\Delta p_{I}}{p_{s}}=\frac{p_{I}-p_{s}}{p_{s}}=\frac{E_{I}-E_{s}}{\beta_{s}^{2} E_{s}}=\frac{\Delta E_{I}}{\beta_{s}^{2} E_{s}} .
$$

The longitudinal emittance of the region is

$$
\epsilon=\left(2 \phi_{I}\right)\left(2 W_{I}\right)=2 \Delta E_{I} T_{s} \frac{\phi_{I}}{\pi} .
$$

\section{RF Capture of a Half Turn}

We consider the case in which a half turn of Au32+ ions from EBIS is injected into Booster. The initial distribution is shown in Figure 6. Here we have taken

$$
\phi_{I}=\frac{\pi}{2}, \quad \frac{\Delta p_{I}}{p_{s}}=0.0005
$$

which gives

$$
W_{I}=0.6366 \mathrm{eVs} .
$$

The fractional momentum half-width is consistent with that obtained from measurements of the turn-by-turn spreading of a short pulse of Au32+ beam injected into Booster with the RF turned off. The longitudinal emittance of the distribution is

$$
\epsilon=4.00 \mathrm{eV} \mathrm{s} .
$$

The barrier bucket in the figure has parameters

$$
V_{B}=2.0 \mathrm{kV}, \quad \Gamma=0.19212 \pi, \quad \lambda=\pi+\Gamma .
$$

The width in time of the barrier bucket pulses is then

$$
T_{s} \Gamma /(2 \pi)=1.0 \mu \mathrm{s} .
$$

To capture the beam in harmonic 4 buckets, the voltage $V_{A}$ is raised from zero to $V_{c}=0.2 \mathrm{kV}$ over capture time $T_{c}=10 \mathrm{~ms}$ according to

$$
V_{A}(T)=V_{c}\left(T / T_{c}\right)^{2}
$$


with the time $T$ going from zero to $T_{c}$. During this time each particle in the distribution is tracked using the turn-by-turn equations of Section 1. The final distribution of particles at time $T_{c}$ is shown in Figure 7. Here we see that all of the beam is cleanly captured into the inner two harmonic 4 buckets. Each of these buckets has area

$$
A_{\mathrm{bk}}=3.00 \mathrm{eV} \mathrm{s} .
$$

If there is no barrier bucket $\left(V_{B}=0\right)$, one obtains the final distribution shown in Figure 8. Here we see that there is leakage of beam into the outer two harmonic 4 buckets.

\section{Squeezing the Beam into a Half Turn}

Consider now the case in which the width of the initial beam distribution is longer than a half turn. In order to end up with all of the beam captured in the inner two harmonic 4 buckets, the barrier bucket must be programmed to squeeze the beam into a half turn before capture. One starts with a barrier bucket that is wide enough to contain the initial distribution. This is shown in Figure 9. Here the width of the initial distribution is 0.8 turns. The fractional momentum half-width is 0.0005 as before. The width of the bucket is then slowly decreased, squeezing the beam down to a half turn in squeeze time $T_{b}$. During this time each particle in the distribution is tracked using the turn-by-turn equations of Section 1. The final distributions for various squeeze times are shown in Figures 10 through 19. Here $T_{b}=2,3,5,7,10,12,15,20,40$ and $80 \mathrm{~ms}$ respectively. Note that although (4) and (5) constitute an area preserving map, there is significant filamentation of the beam distribution if the squeeze time is too short. This is the case for $T_{b}=2,3,5$ and $7 \mathrm{~ms}$. The filamentation is milder for $T_{b}=10,12$ and $15 \mathrm{~ms}$. For $T_{b}=20,40$ and 80 $\mathrm{ms}$ the filamentation is greatly reduced, but these times are too long to be used in practice.

\section{Capturing the Squeezed Beam}

Once the beam distribution has been squeezed into a half turn, capture into the inner two harmonic 4 buckets can take place as described in

Section 7. Figure 20 shows the final distribution obtained after squeezing 
the distribution of Figure 9 to a half turn in $20 \mathrm{~ms}$ and then capturing into harmonic 4 buckets with $T_{c}=10 \mathrm{~ms}$. The harmonic 4 voltage is raised from zero to $V_{c}=0.6 \mathrm{kV}$ over time $T_{c}$ as per equation (57). The beam is cleanly captured into the inner two harmonic 4 buckets. Each of these buckets has area

$$
A_{\mathrm{bk}}=5.20 \mathrm{eV} \mathrm{s} .
$$

In Figure 21 the squeeze time is reduced to $10 \mathrm{~ms}$ with the capture time kept at $10 \mathrm{~ms}$. Here one sees slightly more filamentation in the final beam distribution.

In Figure 22 the squeeze time stays at $10 \mathrm{~ms}$ but the capture time is reduced to $5 \mathrm{~ms}$. Here one sees still more filamentation.

In Figure 23 the squeeze time is reduced further to $5 \mathrm{~ms}$ with the capture time kept at $5 \mathrm{~ms}$. Here there is significant filamentation and a small amount of beam is now outside the harmonic 4 buckets.

In Figure 24 the squeeze time is $6 \mathrm{~ms}$ and the capture time is $6 \mathrm{~ms}$. Here there is slightly less filamentation and the beam is again fully contained in the harmonic 4 buckets.

In Figure 25 the squeeze time is $7 \mathrm{~ms}$ and the capture time is $5 \mathrm{~ms}$. Here the beam has slightly less energy spread in the buckets.

These figures show that in order for the beam to be contained in buckets with area given by (59), the squeeze time needs to be at least $6 \mathrm{~ms}$ and the combined squeeze and capture time needs to be at least $12 \mathrm{~ms}$.

\section{Estimate of Longitudinal Emittance in AGS}

After transfer of 8 loads of 2 bunches from Booster into 16 buckets on the AGS injection porch, the 16 bunches are merged into 4. The minimum longitudinal emittance per merged bunch is then

$$
A_{\text {min }}=4 A_{\mathrm{bk}}
$$

where $A_{\mathrm{bk}}$ is the bucket area at the end of capture in Booster. As calculated in Section 7, $A_{\mathrm{bk}}=3.00 \mathrm{eV} \mathrm{s}$ for the case in which a half turn of Au32+ beam is injected from EBIS. If instead 0.8 turn is injected and squeezed to a half turn, then $A_{\mathrm{bk}}$ increases to $5.20 \mathrm{eV} \mathrm{s}$ as calculated in Section 9. The estimated longitudinal emittance per merged bunch is

$$
A_{\mathrm{est}}=K A_{\mathrm{min}}=4 K A_{\mathrm{bk}}
$$


where the factor $K$ depends on the emittance increases during acceleration in Booster, upon passage through the BTA stripping foil, and during the bunch merging on the AGS injection porch. If we take increase factors of 1.5 and 1.8 for the first two processes and a factor of 1.2 for each of two bunch merges (16 bunches are merged to 8 and then to 4 ), then

$$
K=1.5 \times 1.8 \times 1.2 \times 1.2=3.888
$$

and

$$
A_{\mathrm{est}}=15.552 A_{\mathrm{bk}} \text {. }
$$

Taking $A_{\mathrm{bk}}=3.00 \mathrm{eV}$ s then gives $A_{\mathrm{est}}=46.656 \mathrm{eVs}$ which amounts to $46.656 / 197=0.237 \mathrm{eV}$ s per nucleon.

Taking $A_{\mathrm{bk}}=5.20 \mathrm{eV}$ s then gives $A_{\mathrm{est}}=80.8704 \mathrm{eV} \mathrm{s}$ which amounts to $80.8704 / 197=0.4105 \mathrm{eV}$ s per nucleon.

\section{References}

[1] K.L. Zeno, Booster-AGS-EBIS-2012 elog.

[2] C.J. Gardner, "RF Capture Simulation for Gold Ions in AGS", C-A/AP Note 29, October 2000

[3] J.A. MacLachlan, "Difference Equations for Longitudinal Motion in a Synchrotron", Fermilab internal report FNAL FN-529, December 15, 1989; "Differential Equations for Longitudinal Motion in a Synchrotron", Fermilab internal report FNAL FN-532, January 25, 1990.

[4] H. Yoshida, Numerical Integration Methods, Handbook of Accelerator Physics and Engineering, Edited by A.W. Chao and M. Tigner, World Scientific, 1999, pp. 85-87. 


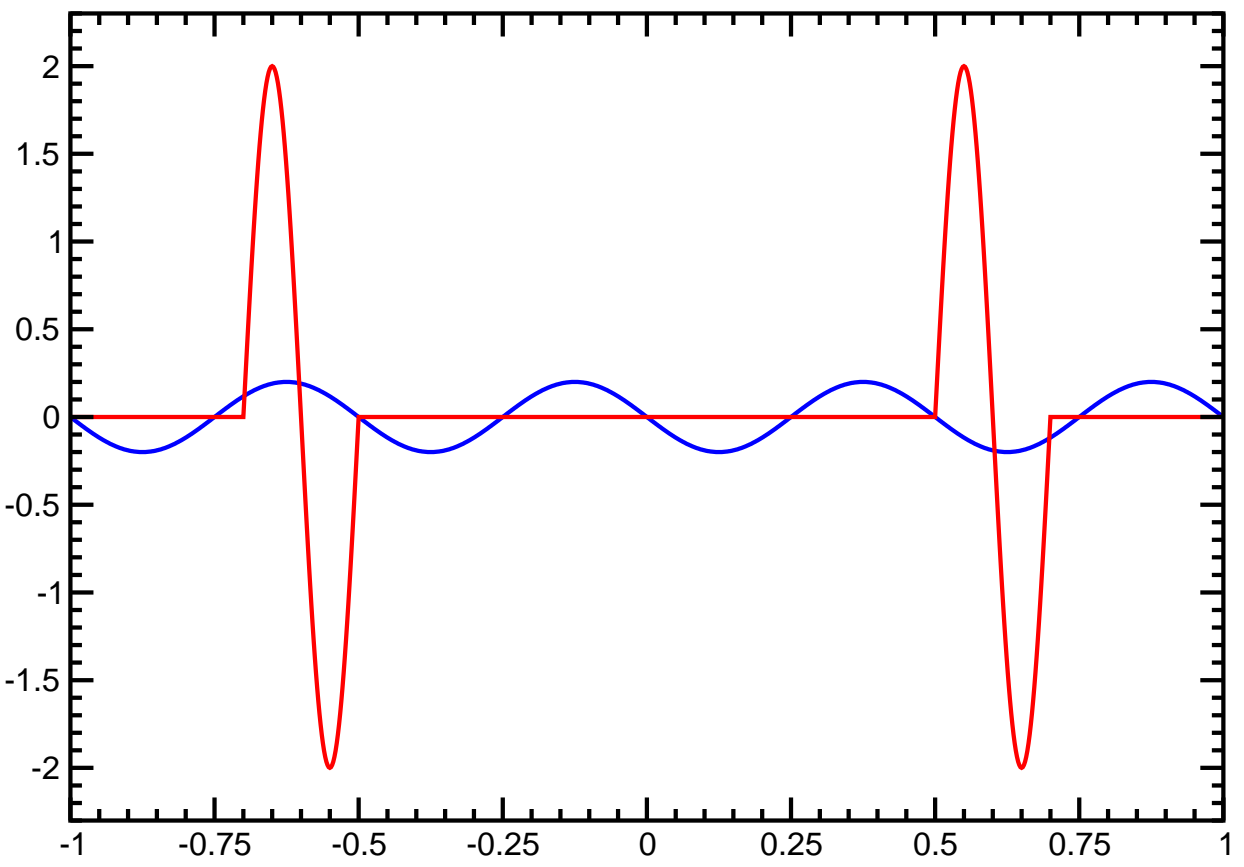

Figure 1: Excitation of the harmonic 4 and barrier bucket cavities. The blue and red curves are respectively the harmonic 4 excitation, $-A \sin 4 \phi$, and the barrier bucket excitation $\Delta(\phi) B \sin \chi$. Here we have taken $A=0.2$, $B=2.0, \Gamma=0.2 \pi$, and $\lambda=\pi+\Gamma$. The horizontal axis is $\phi / \pi$. Note that the region of zero voltage between the two barrier pulses starts and ends where the harmonic 4 voltage crosses zero. This allows a half turn of beam to be held in place by the barrier bucket and be captured into two harmonic 4 buckets as the harmonic 4 voltage is raised from zero. 


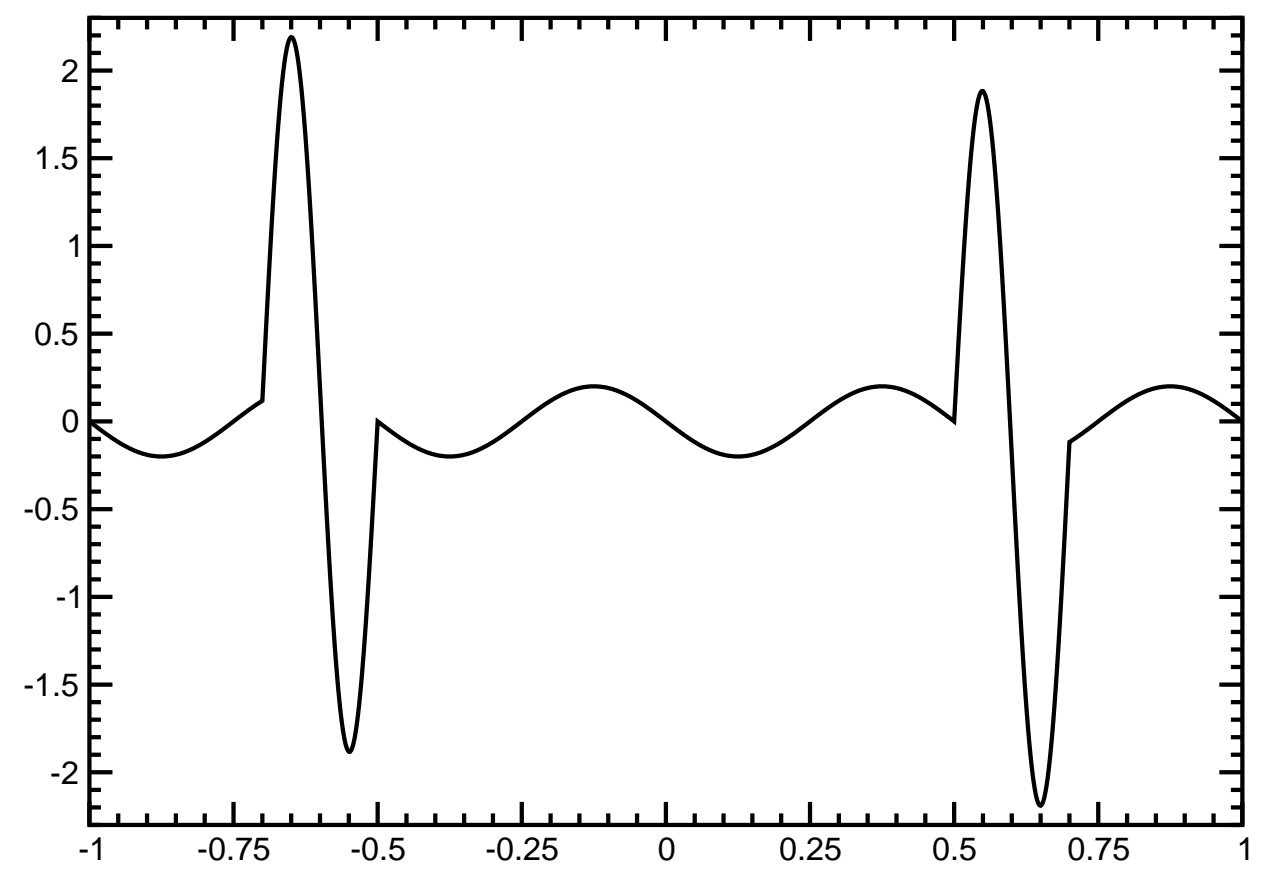

Figure 2: This is the sum, $F(\phi)=\Delta(\phi) B \sin \chi-A \sin 4 \phi$, of the red and blue curves in Figure 1. The horizontal axis is $\phi / \pi$. 


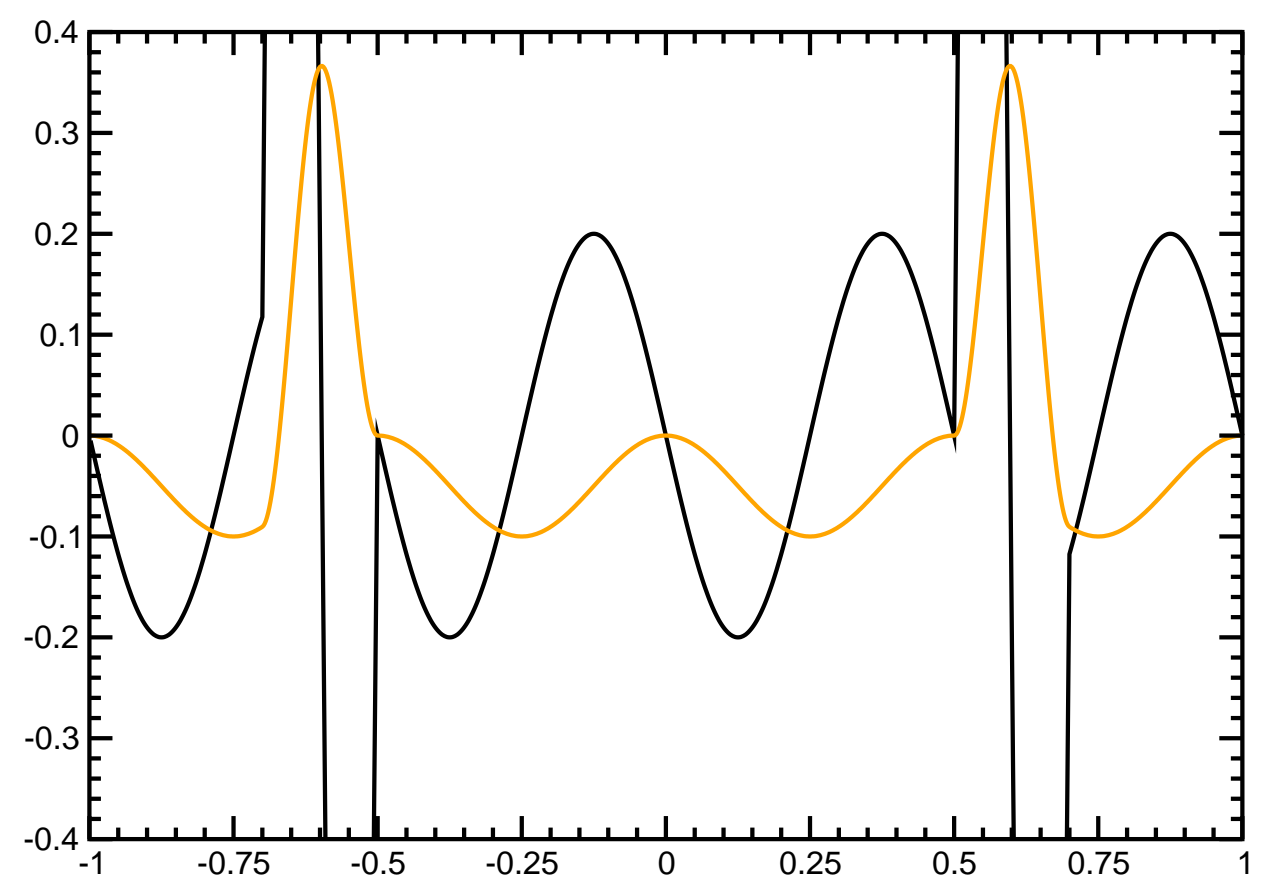

Figure 3: Here the black curve is $F(\phi)$ from Figure 2. The orange curve is the corresponding $-U(\phi)$. The largest maxima of the orange curve occur at $\phi= \pm 0.5970 \pi$. These are unstable fixed points. The points $\phi=$ $0, \pm \pi / 2, \pm \pi$ are also unstable fixed points. The points $\phi= \pm \pi / 4, \pm 3 \pi / 4$ are stable fixed points. The horizontal axis is $\phi / \pi$. 


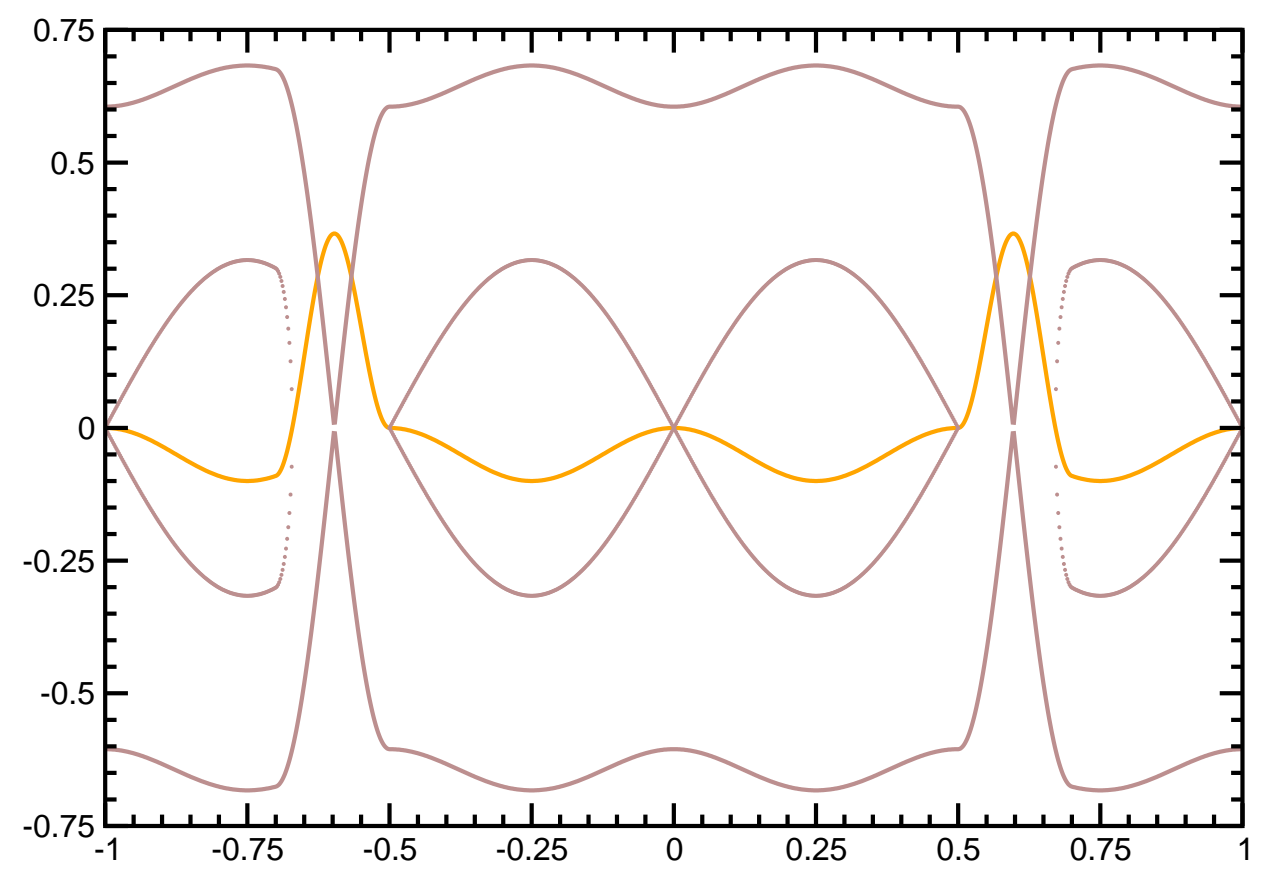

Figure 4: Here the orange curve is $-U(\phi)$ from Figure 3. The outer brown curves are the separatrices that pass through unstable fixed points $\phi_{u}= \pm 0.5970 \pi$. These curves form the barrier bucket. The inner brown curves are the separatrices that pass through the unstable fixed points $\phi_{u}=0, \pm \pi / 2, \pm \pi$. These curves form the harmonic 4 buckets. Note that the barrier bucket is distorted by the harmonic 4 voltage, as are two (the left-most and right-most) of the harmonic 4 buckets by the barrier bucket voltage. The horizontal axis is $\phi / \pi$. 


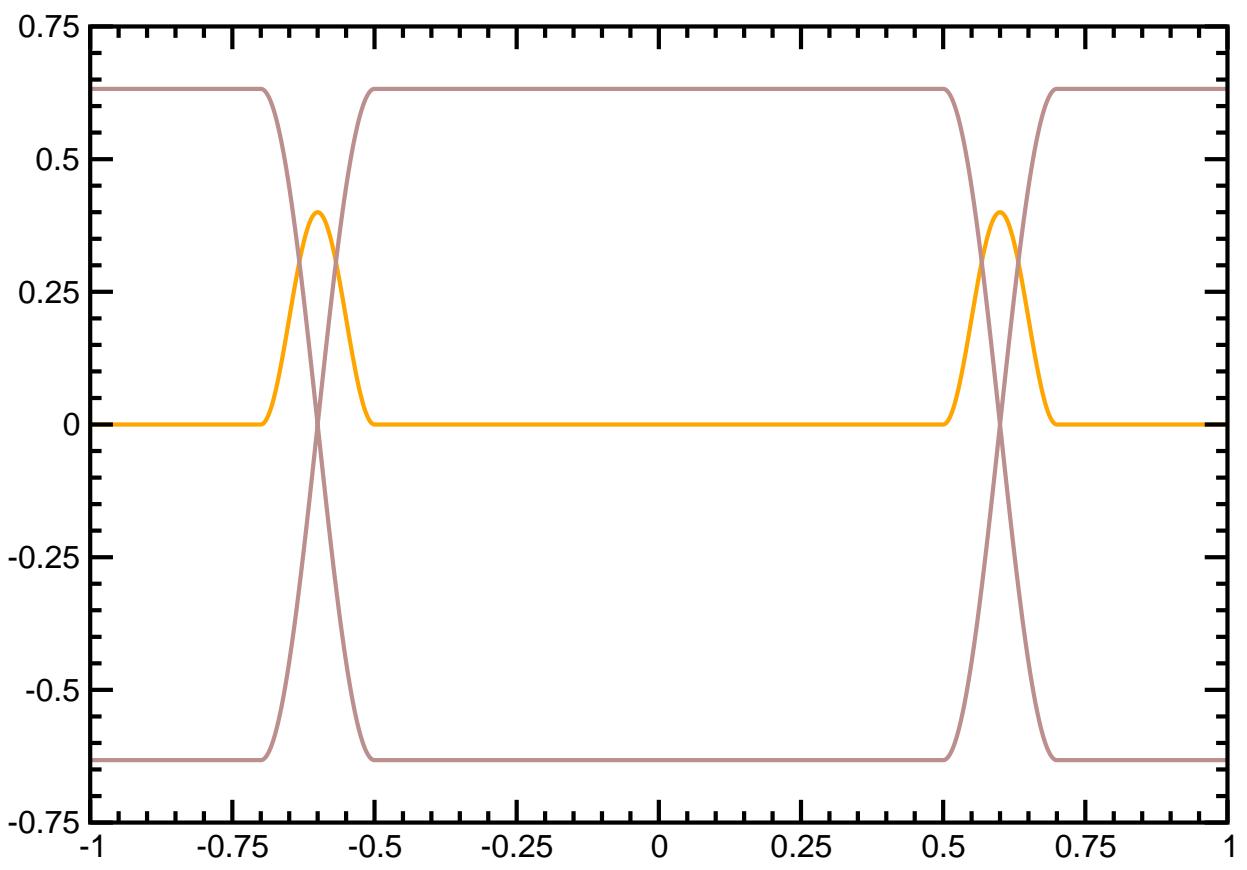

Figure 5: Barrier bucket with no harmonic 4 voltage $\left(V_{A}=0\right)$. 


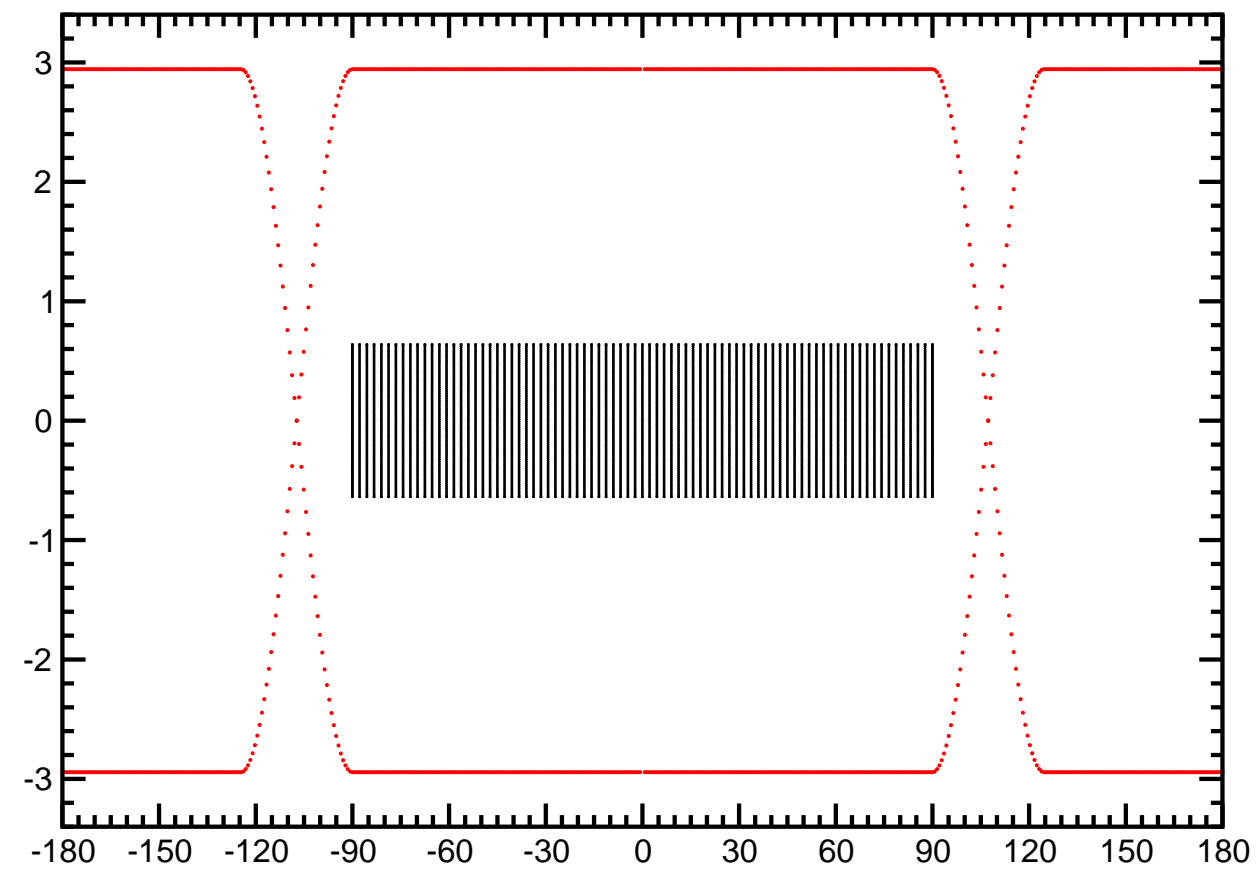

Figure 6: Initial half turn particle distribution in barrier bucket. Here the horizontal axis is the phase $\phi$ in degrees; the vertical axis is $W$ in units of eVs. The half-width of the distribution is $\phi_{I}=\pi / 2$. The fractional momentum half-width is $\Delta p_{I} / p_{s}=0.0005$ which gives $W_{I}=0.6366 \mathrm{eV} \mathrm{s}$. The longitudinal emittance of the distribution is $4.00 \mathrm{eV} \mathrm{s}$. The parameters of the barrier bucket are $V_{B}=2.0 \mathrm{kV}, \Gamma=0.19212 \pi$ and $\lambda=\pi+\Gamma$. The width in time of the barrier bucket pulses is $T_{s} \Gamma /(2 \pi)=1.0 \mu \mathrm{s}$. 


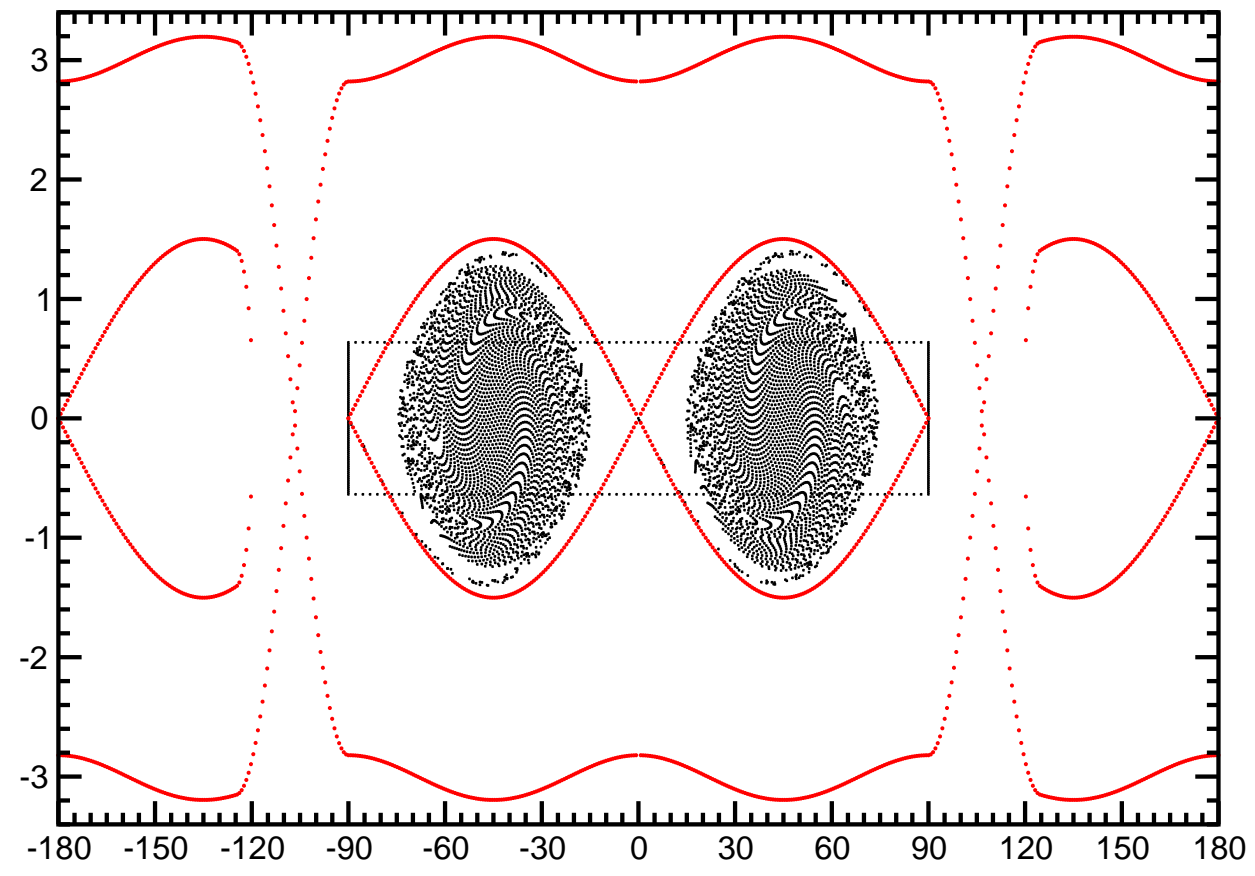

Figure 7: Capture of the half turn distribution of Figure 6 into two harmonic 4 buckets. Here, again, the horizontal axis is the phase $\phi$ in degrees; the vertical axis is $W$ in units of eV s. The black rectangle shows the border of the initial distribution. The harmonic 4 voltage is raised from zero to $V_{c}=0.2 \mathrm{kV}$ over time $T_{c}=10 \mathrm{~ms}$ as per equation (57). The beam is cleanly captured into the inner two harmonic 4 buckets. Each of these buckets has area $A_{\mathrm{bk}}=3.00 \mathrm{eV} \mathrm{s}$. 


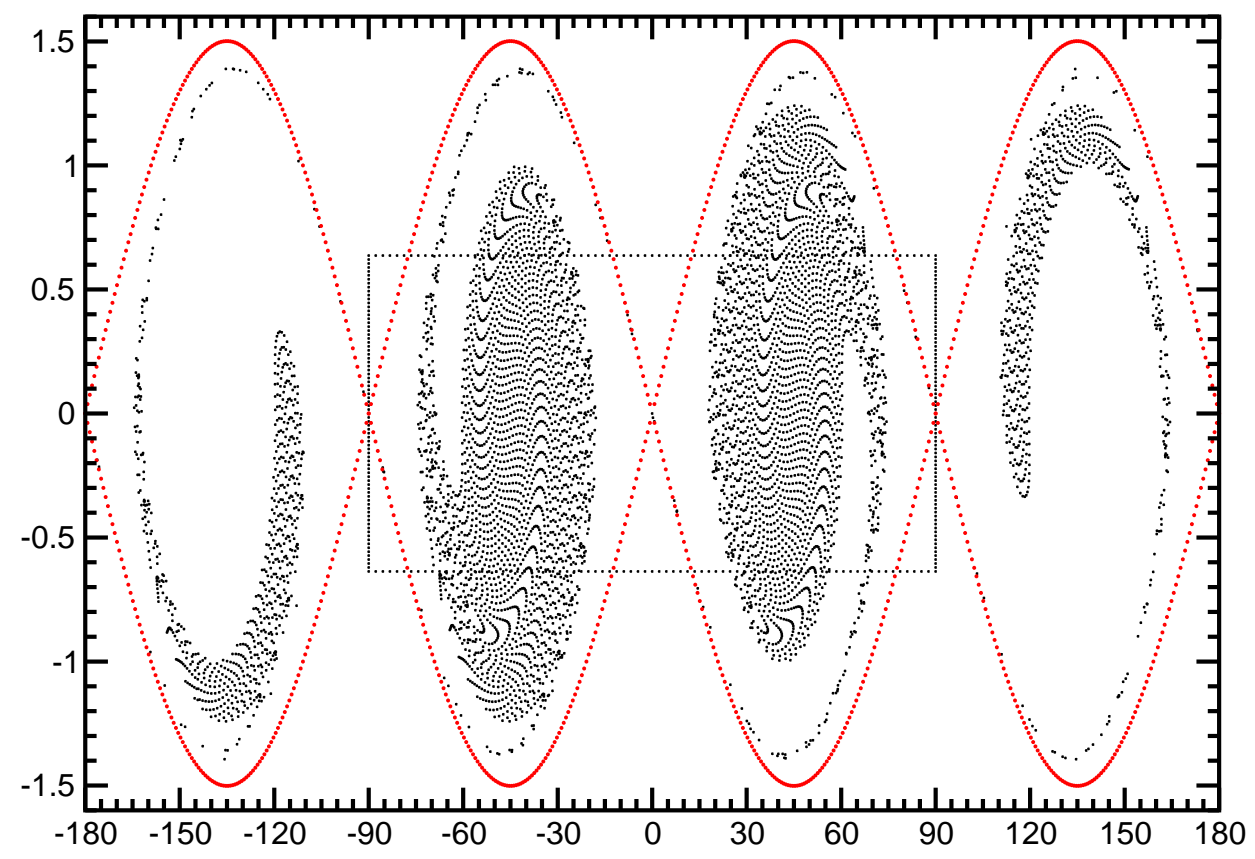

Figure 8: Capture of half turn with no barrier bucket $\left(V_{B}=0\right)$. Here we see that there is leakage of beam into the outer two harmonic 4 buckets. 


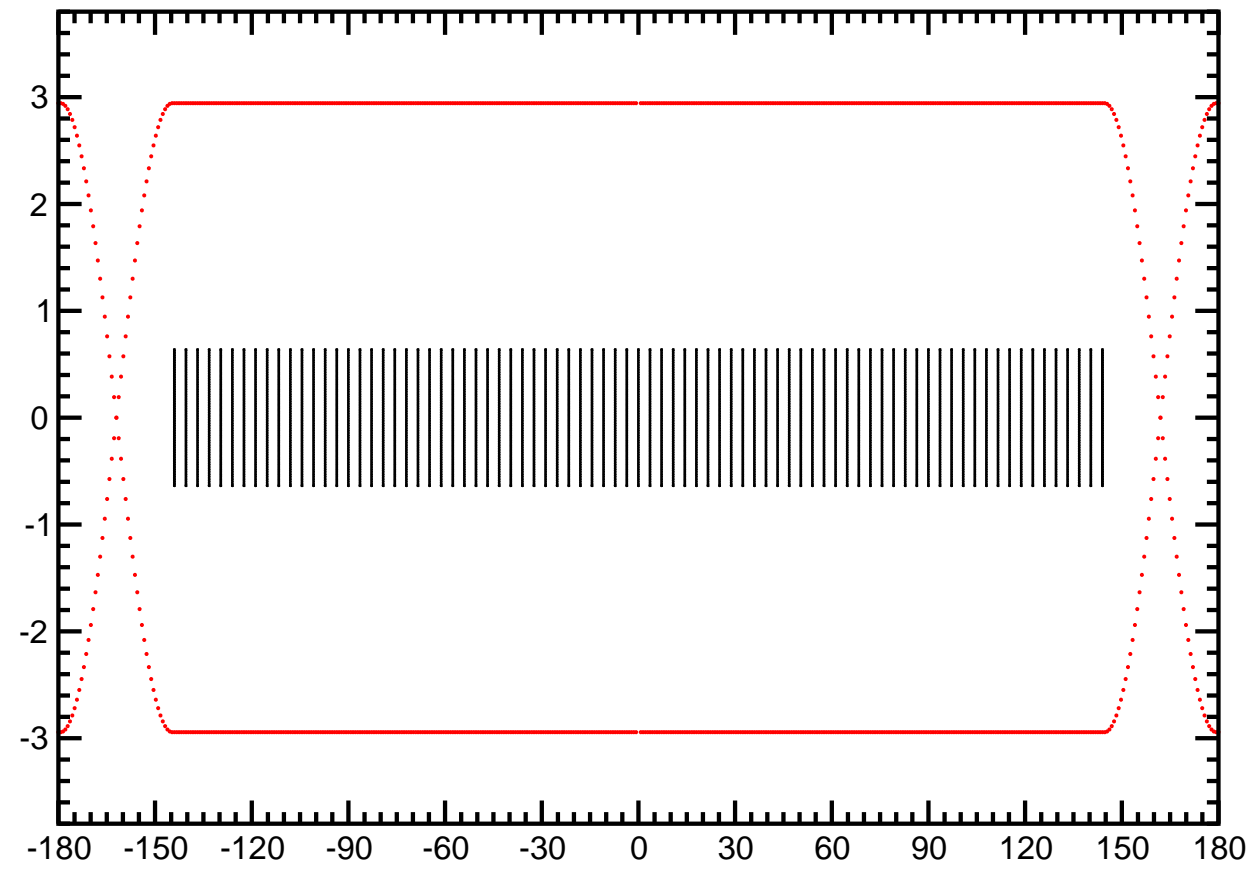

Figure 9: Initial 0.8 turn particle distribution in barrier bucket. Here as before the horizontal axis is the phase $\phi$ in degrees; the vertical axis is $W$ in units of eV s. The half-width of the distribution is $\phi_{I}=0.8 \pi$. The fractional momentum half-width is $\Delta p_{I} / p_{s}=0.0005$ which gives $W_{I}=0.6366 \mathrm{eV} \mathrm{s}$. The longitudinal emittance of the distribution is $6.40 \mathrm{eV} \mathrm{s}$. The parameters of the barrier bucket are $V_{B}=2.0 \mathrm{kV}, \Gamma=0.19212 \pi$ and $\lambda=1.8 \pi$. The width in time of the barrier bucket pulses is $T_{s} \Gamma /(2 \pi)=1.0 \mu \mathrm{s}$. 


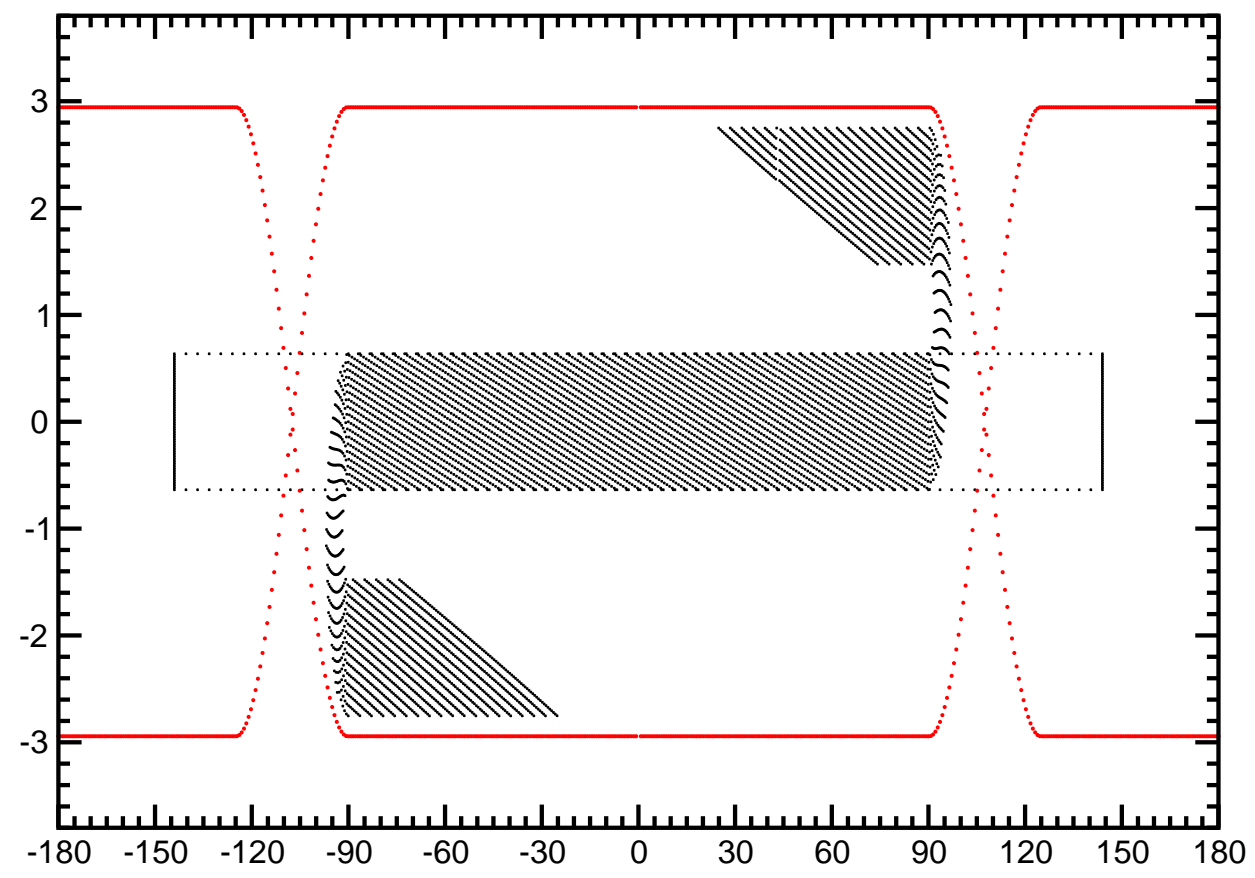

Figure 10: Squeeze of Figure 9 distribution to half turn in $2 \mathrm{~ms}$. 


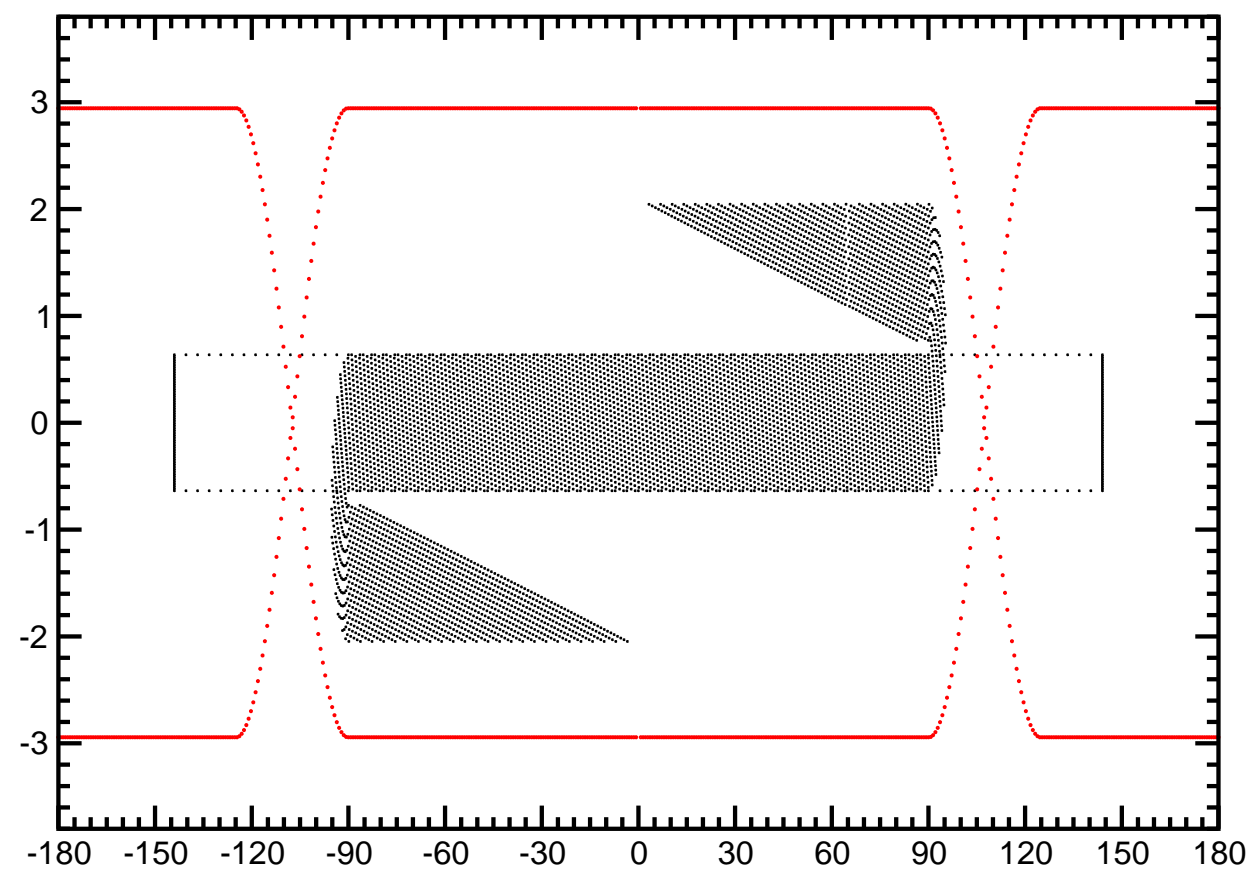

Figure 11: Squeeze to half turn in $3 \mathrm{~ms}$. 


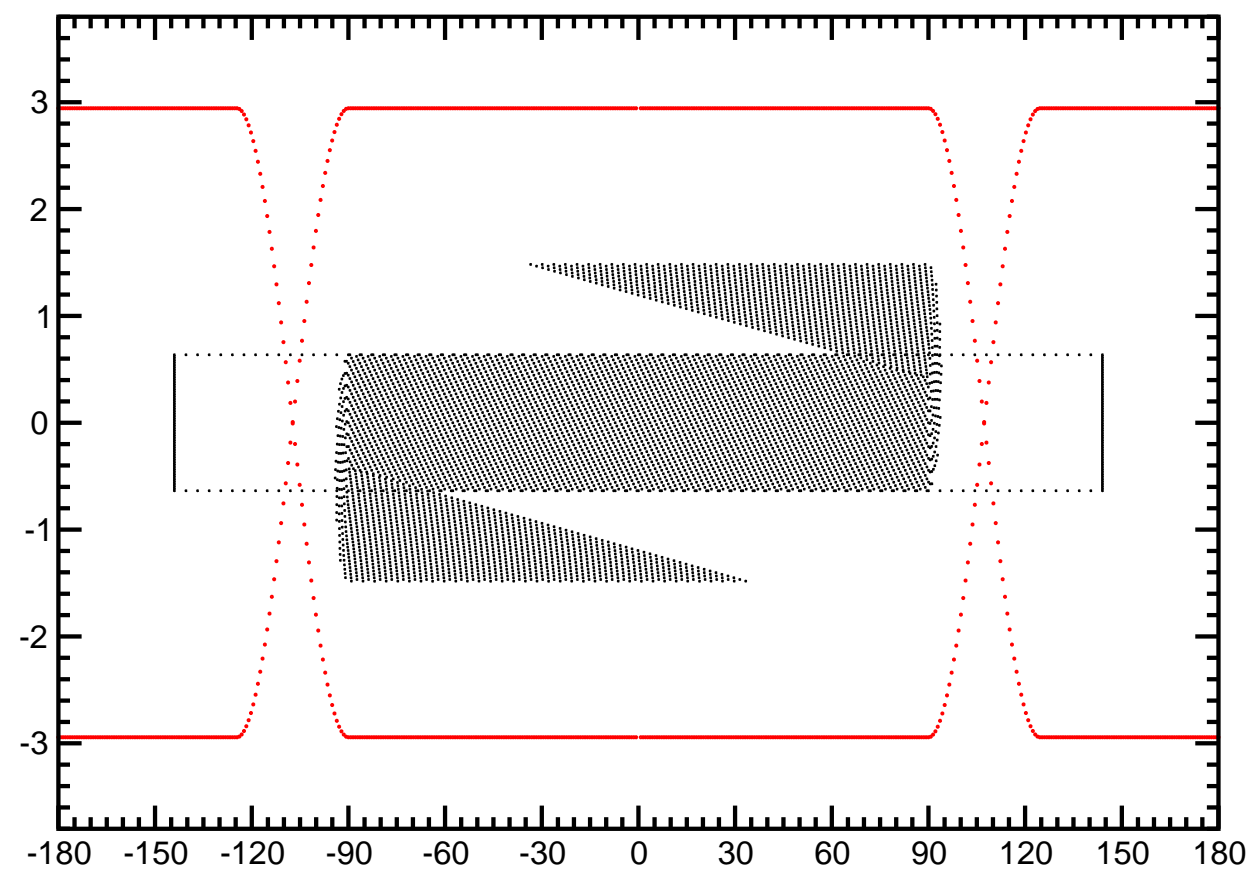

Figure 12: Squeeze to half turn in $5 \mathrm{~ms}$. 


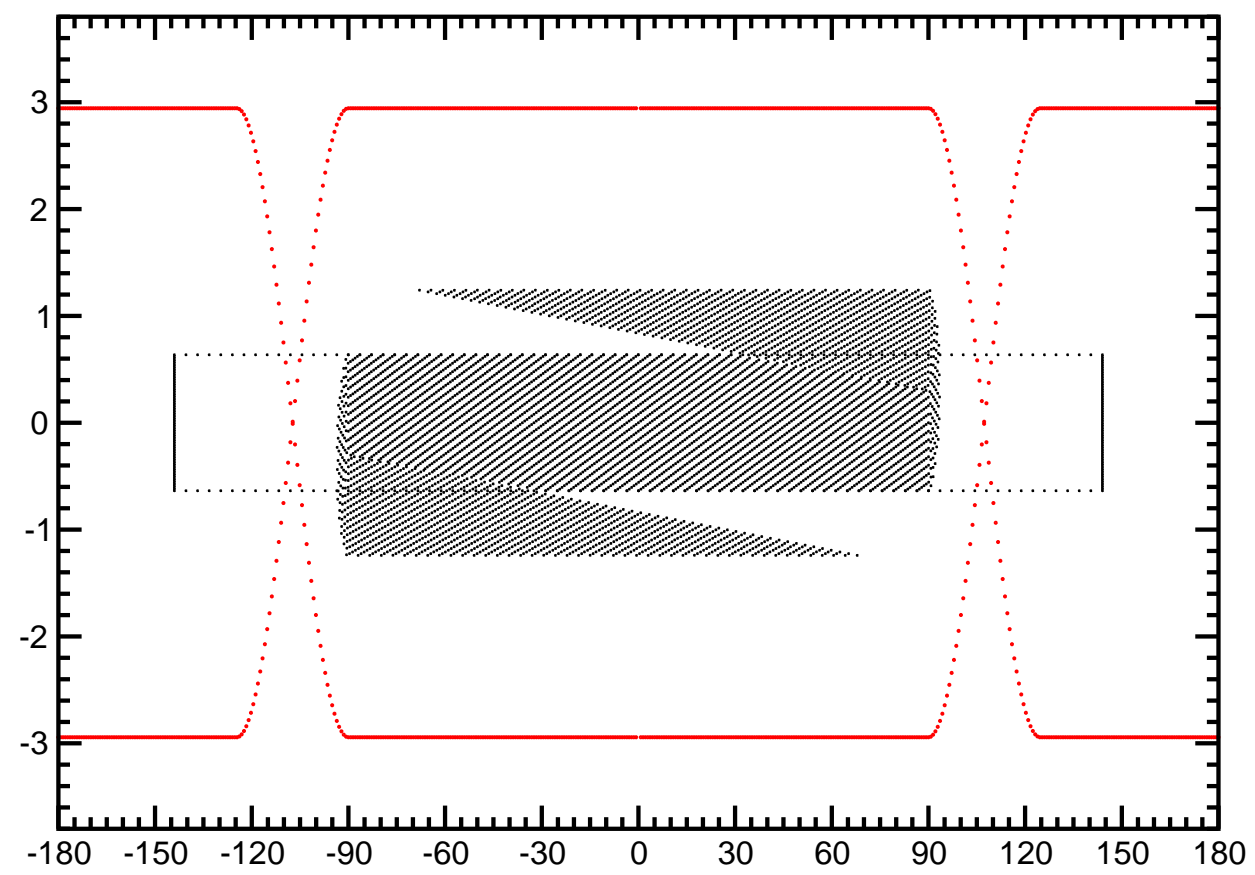

Figure 13: Squeeze to half turn in $7 \mathrm{~ms}$. 


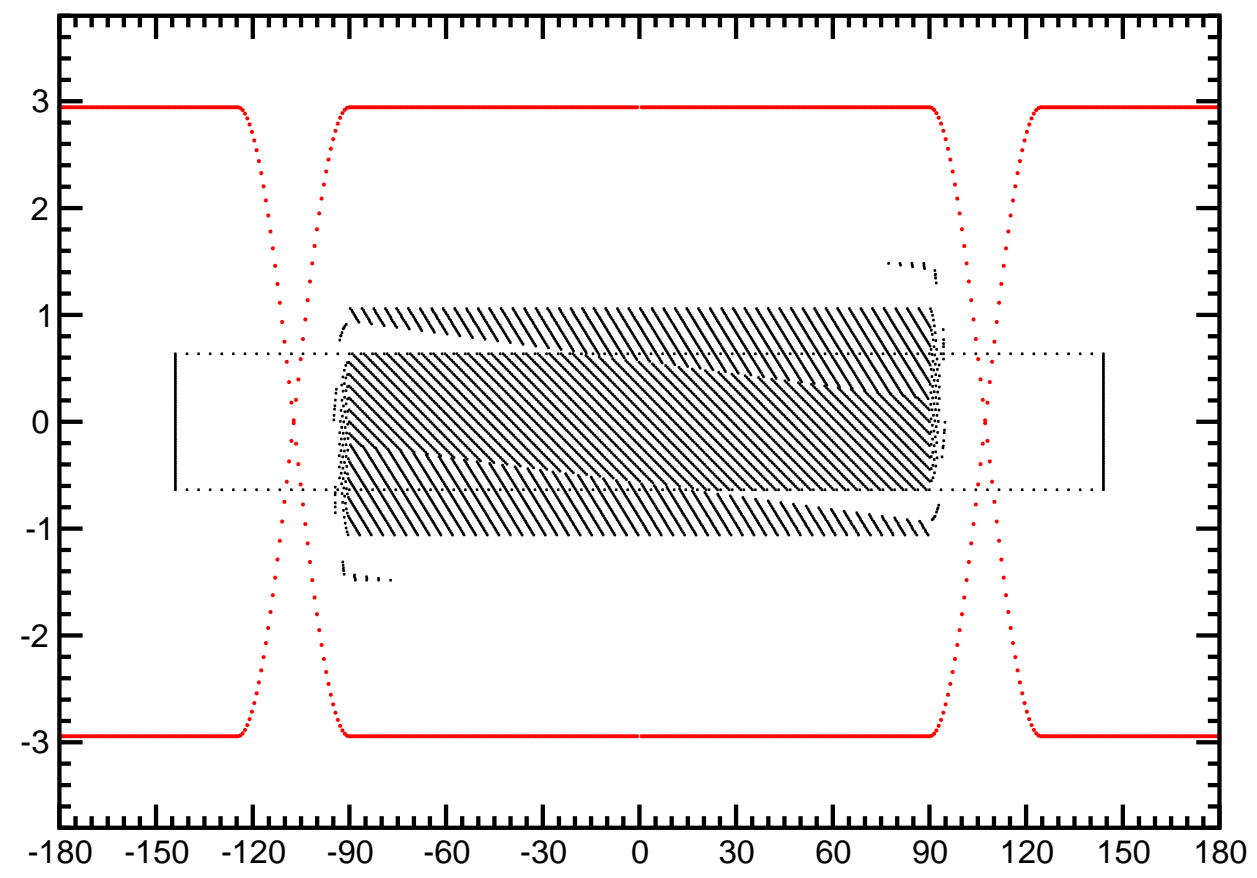

Figure 14: Squeeze to half turn in $10 \mathrm{~ms}$. 


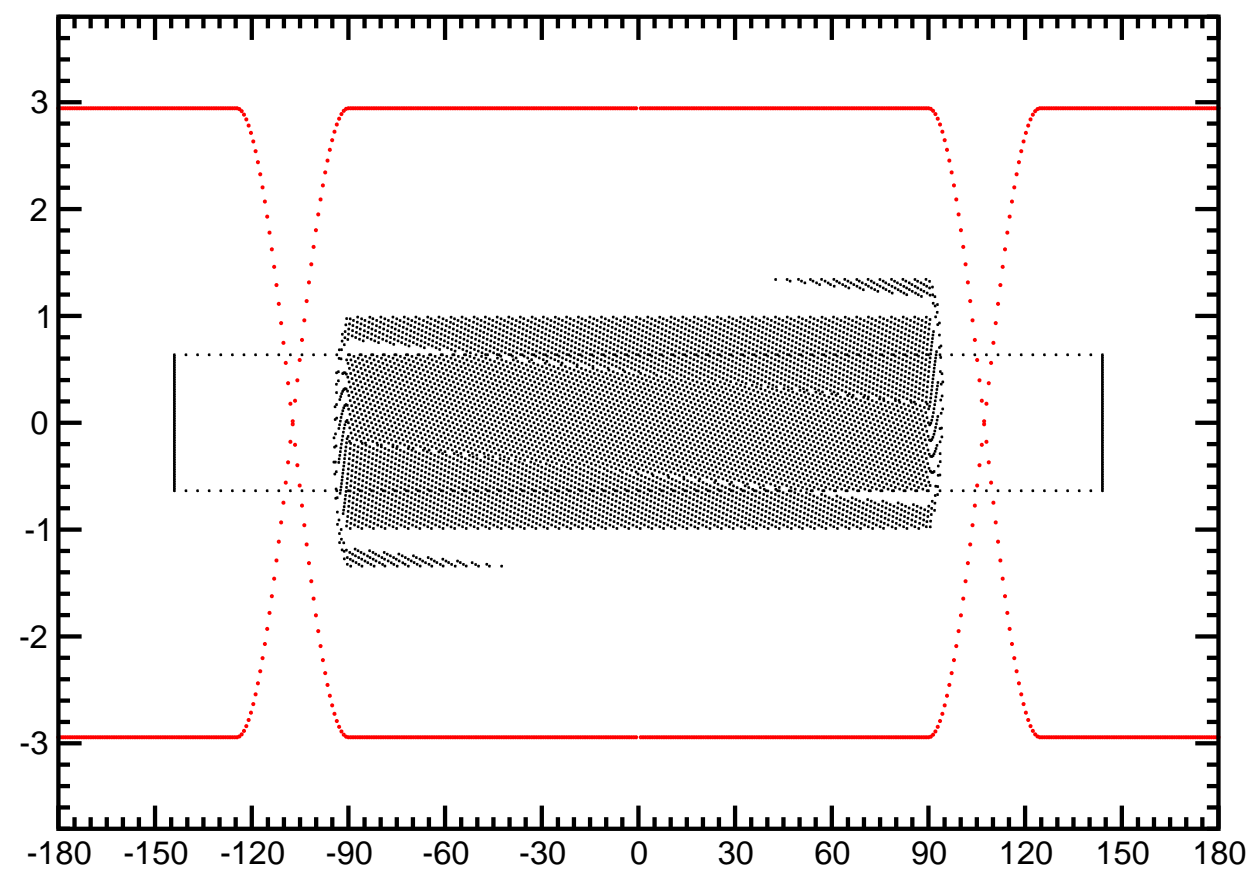

Figure 15: Squeeze to half turn in $12 \mathrm{~ms}$. 


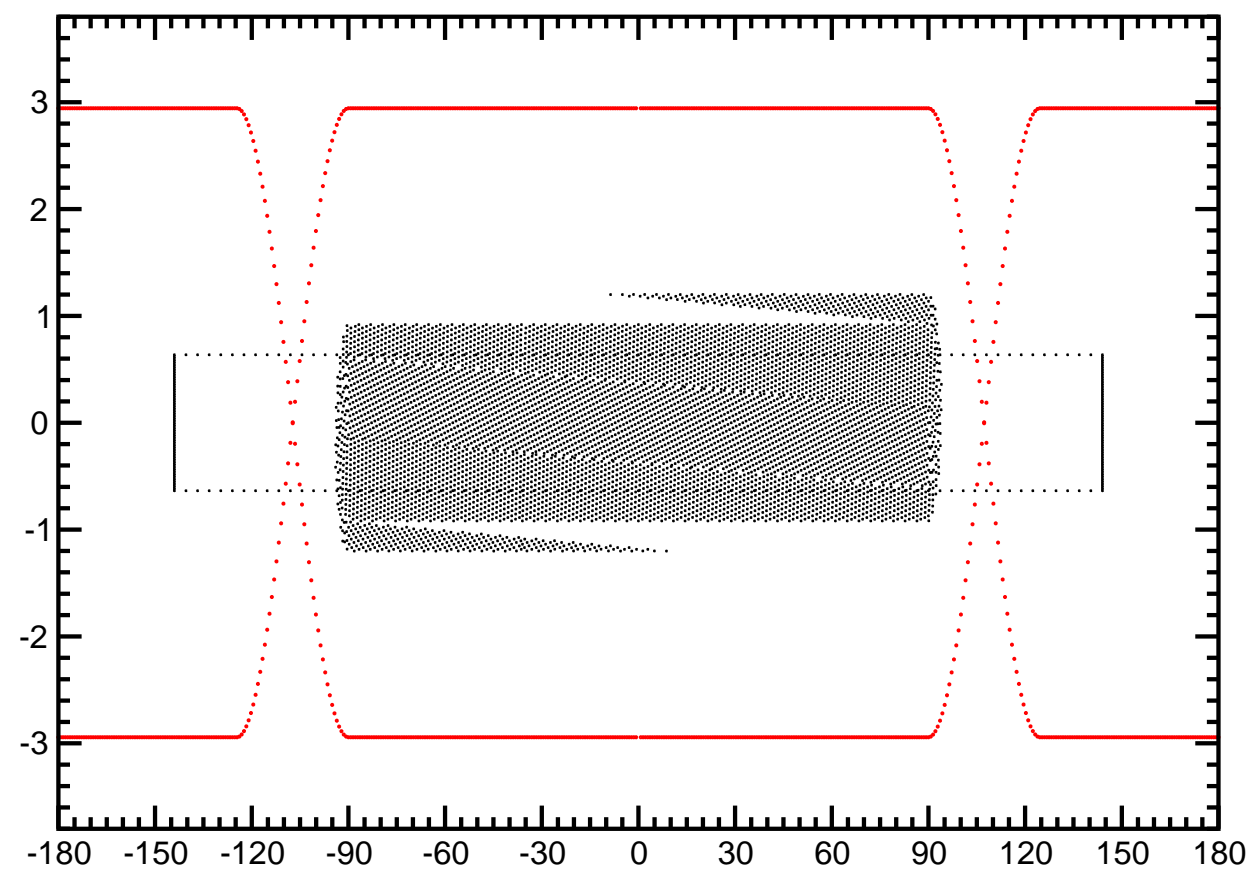

Figure 16: Squeeze to half turn in $15 \mathrm{~ms}$. 


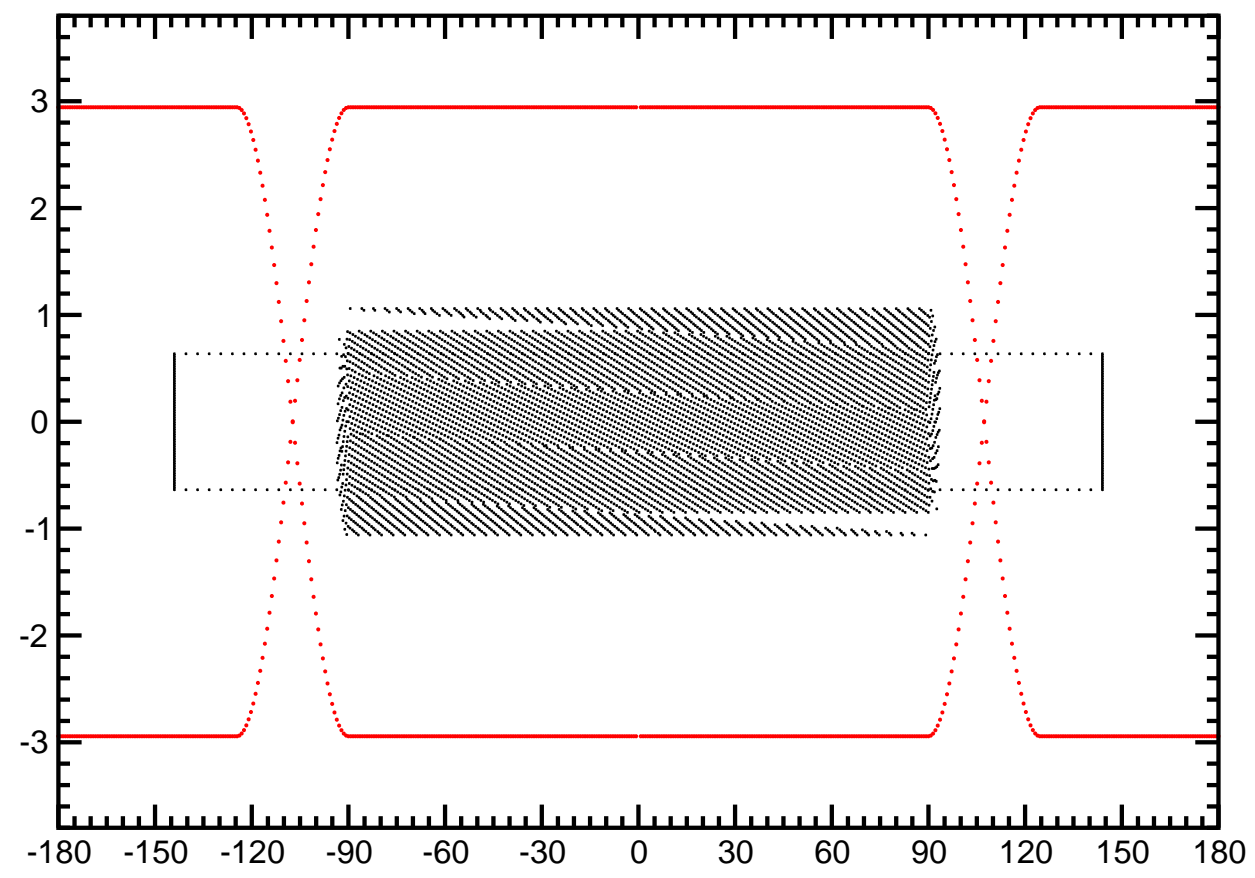

Figure 17: Squeeze to half turn in $20 \mathrm{~ms}$. 


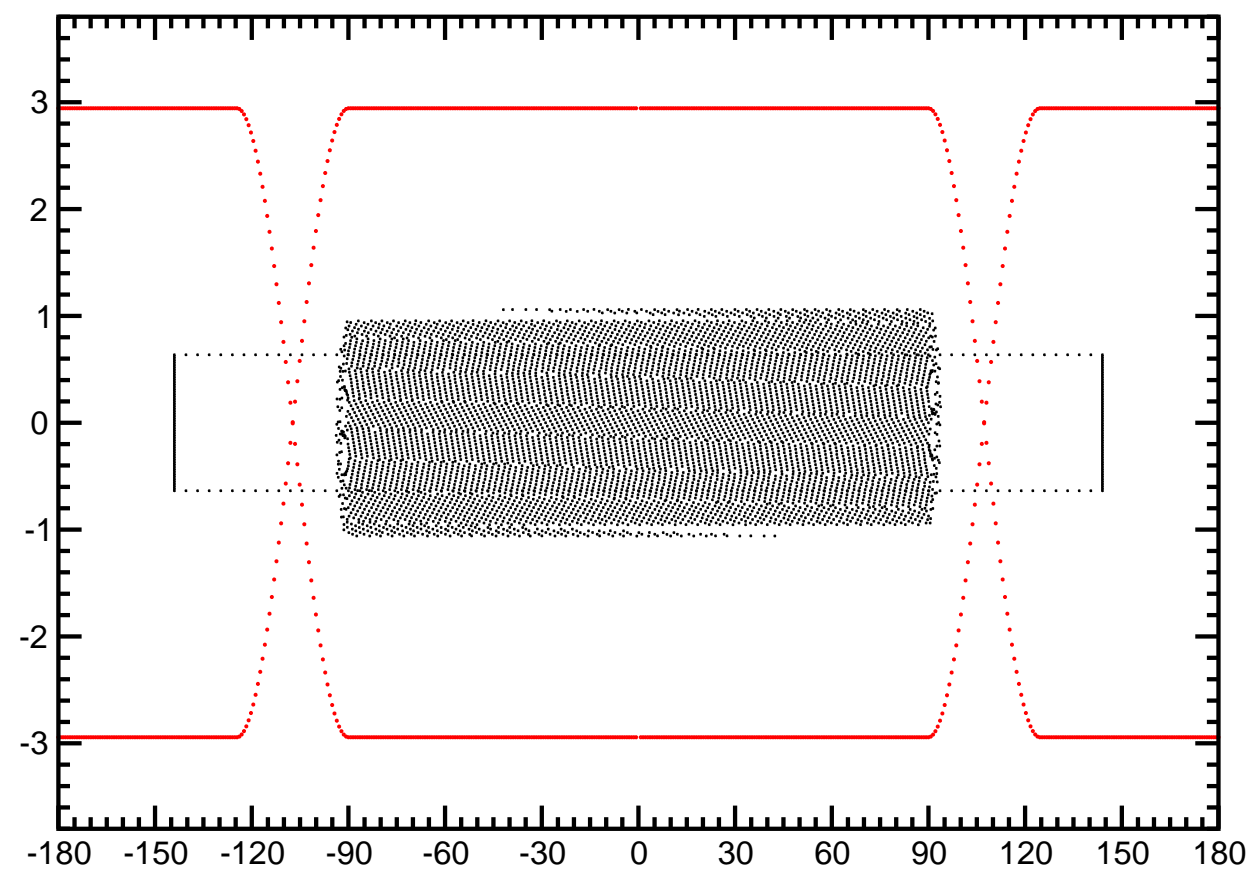

Figure 18: Squeeze to half turn in $40 \mathrm{~ms}$. 


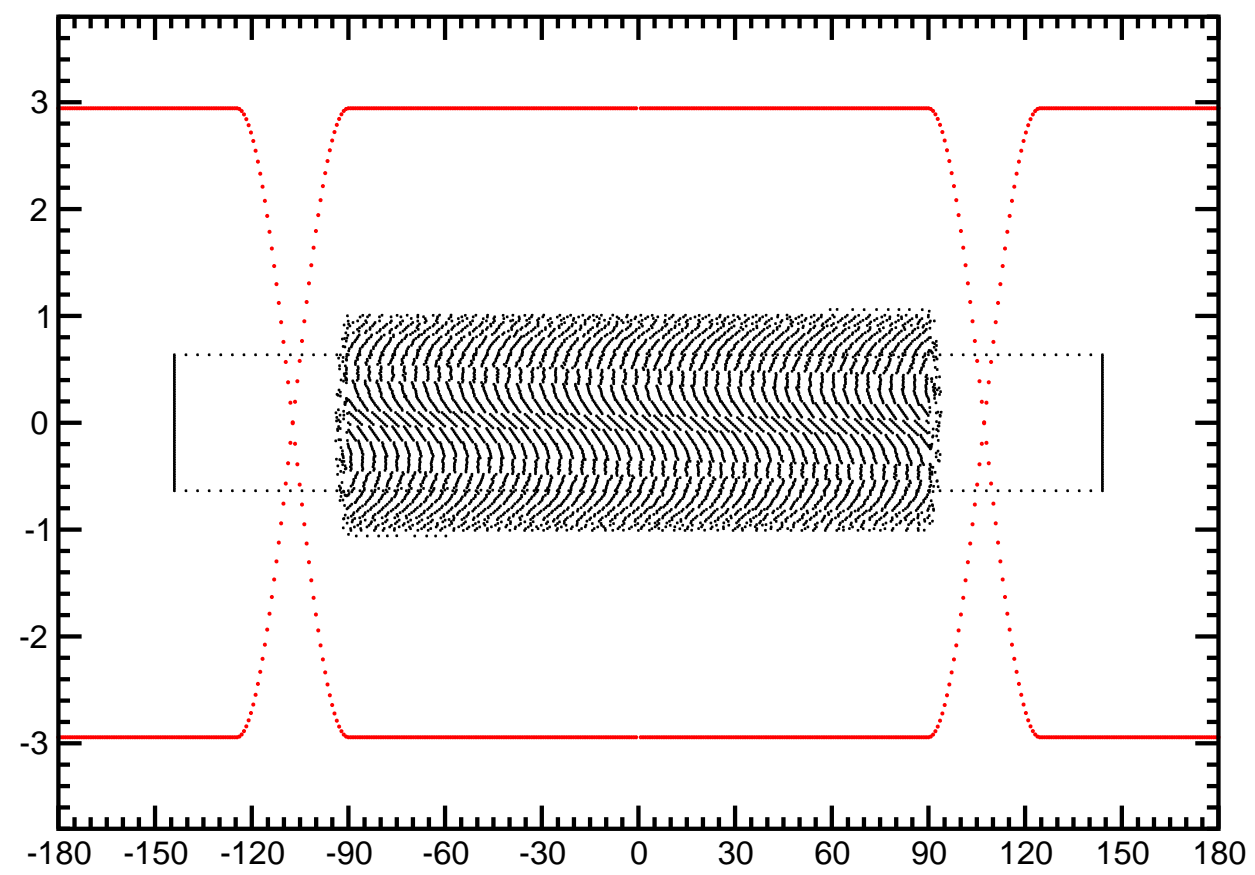

Figure 19: Squeeze to half turn in $80 \mathrm{~ms}$. 


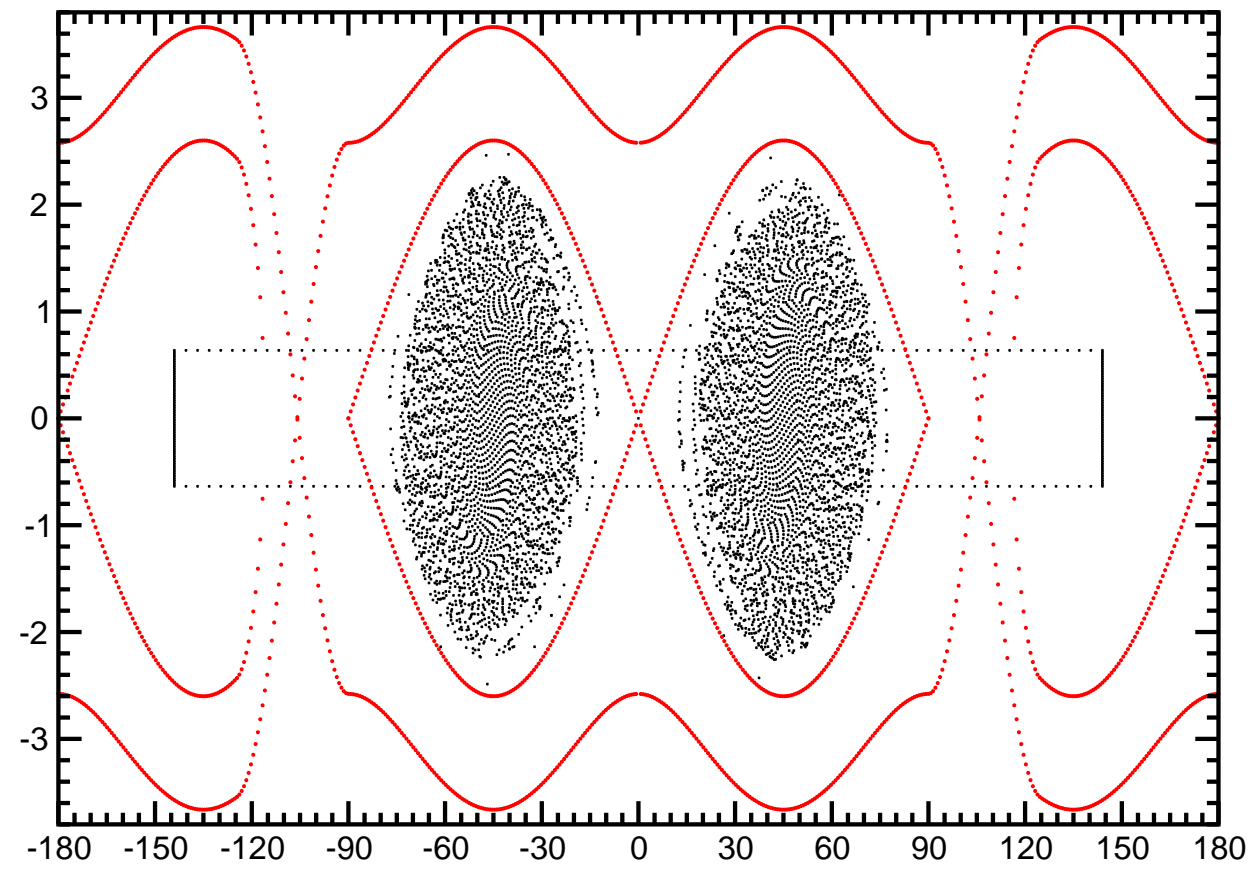

Figure 20: Squeeze of Figure 9 distribution to half turn in $20 \mathrm{~ms}$ followed by harmonic 4 capture with $T_{c}=10 \mathrm{~ms}$. Here as before the horizontal axis is the phase $\phi$ in degrees; the vertical axis is $W$ in units of eVs. The black rectangle shows the border of the initial distribution. The harmonic 4 voltage is raised from zero to $V_{c}=0.6 \mathrm{kV}$ over time $T_{c}$ as per equation (57). The beam is cleanly captured into the inner two harmonic 4 buckets. Each of these buckets has area $A_{\mathrm{bk}}=5.20 \mathrm{eV} \mathrm{s}$. 


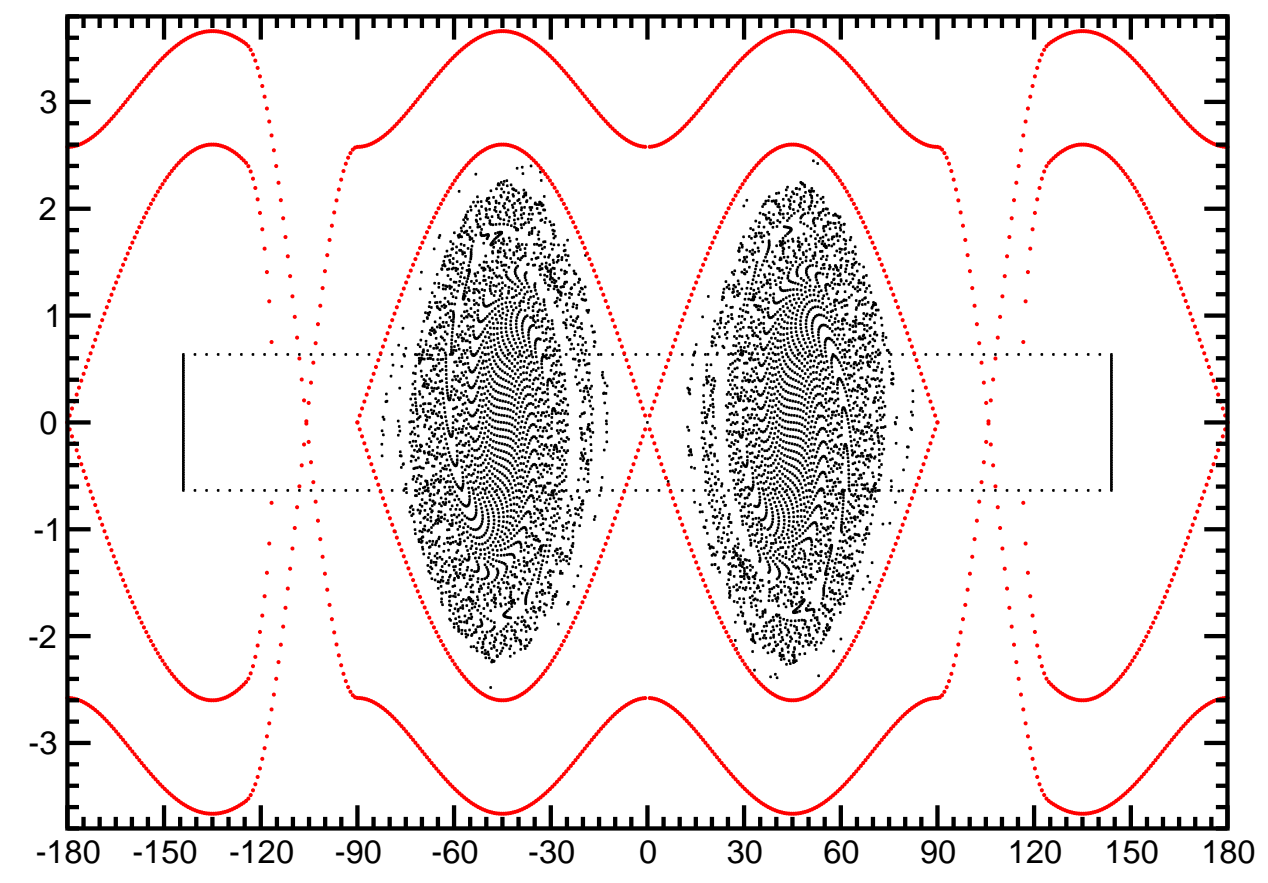

Figure 21: Same as Figure 20 but with $10 \mathrm{~ms}$ squeeze to half turn followed by $10 \mathrm{~ms}$ harmonic 4 capture. 


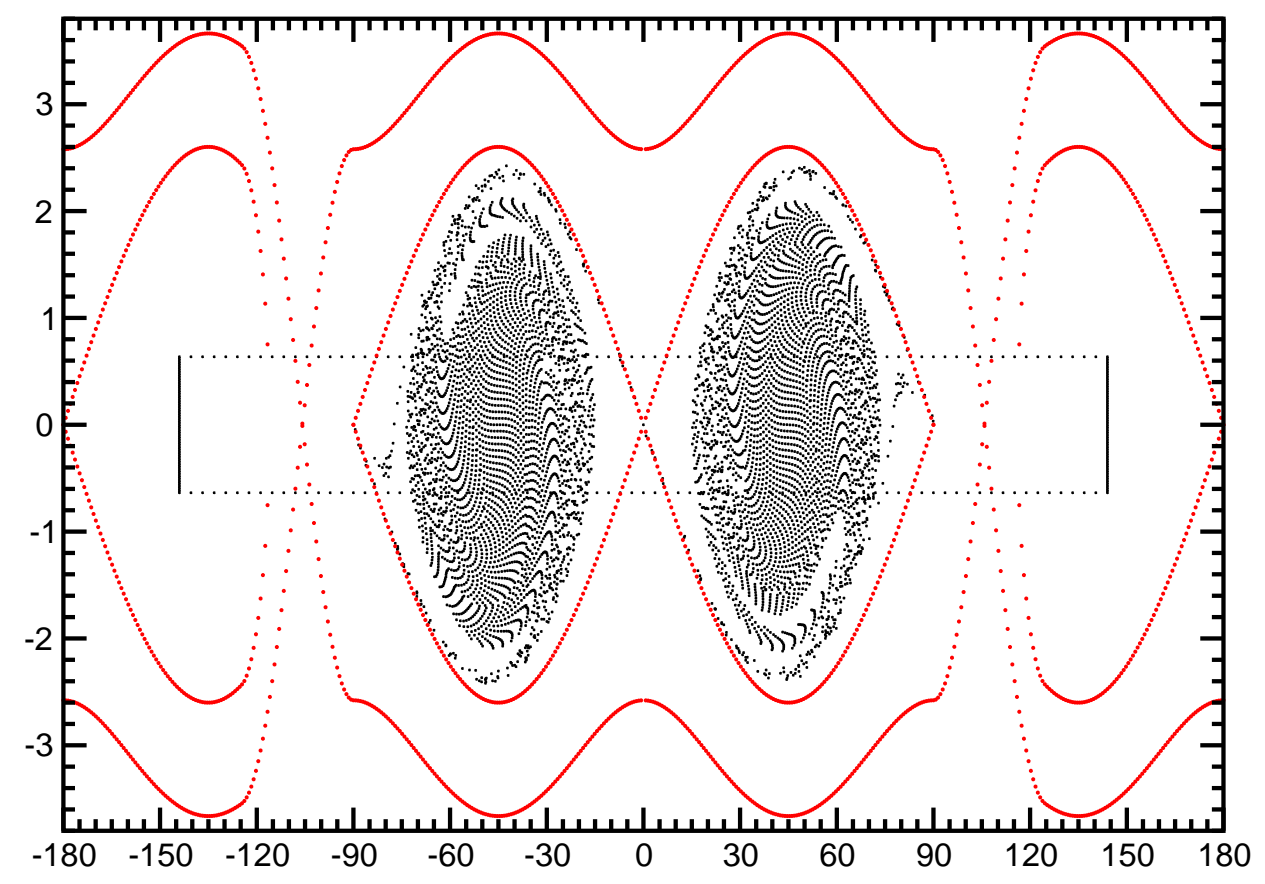

Figure 22: $10 \mathrm{~ms}$ squeeze to half turn followed by $5 \mathrm{~ms}$ harmonic 4 capture. 


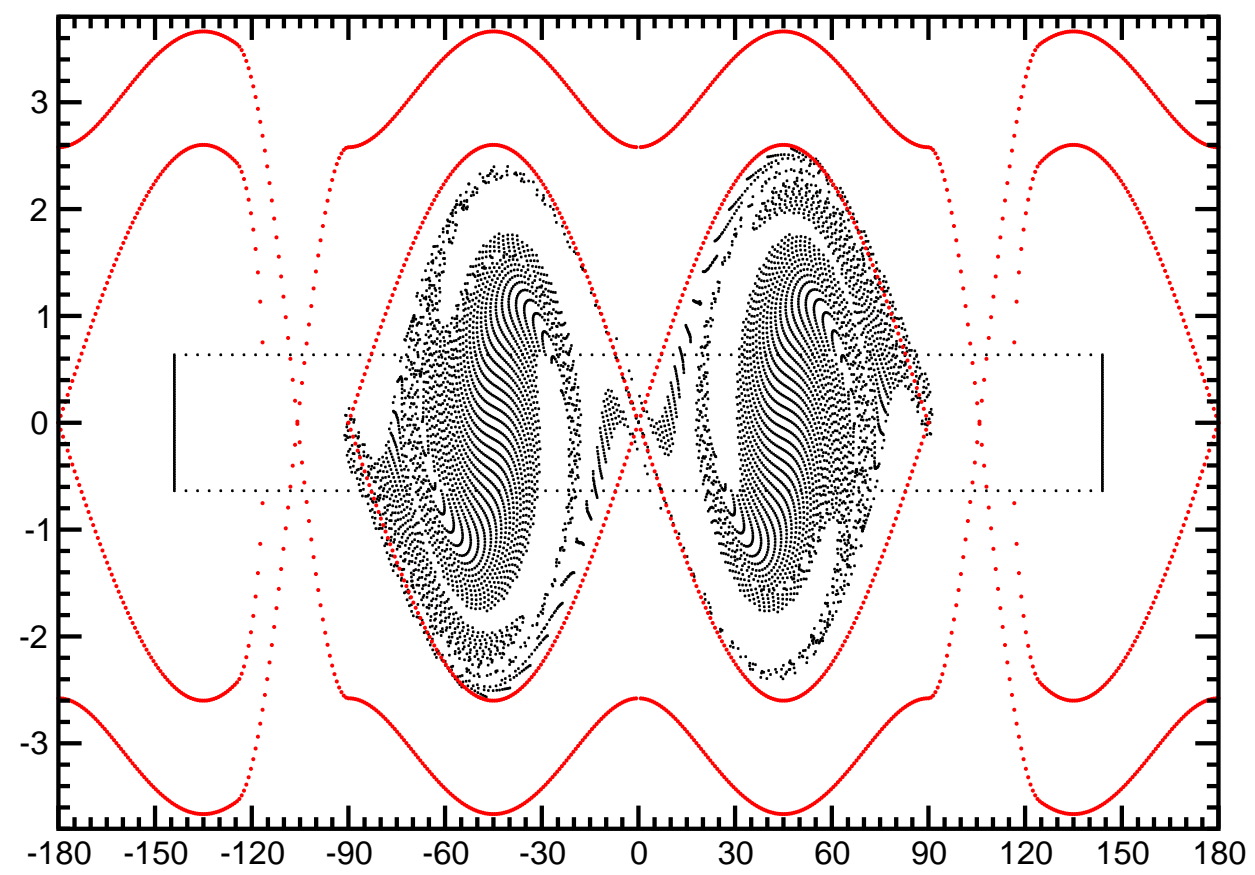

Figure 23: $5 \mathrm{~ms}$ squeeze to half turn followed by $5 \mathrm{~ms}$ harmonic 4 capture. 


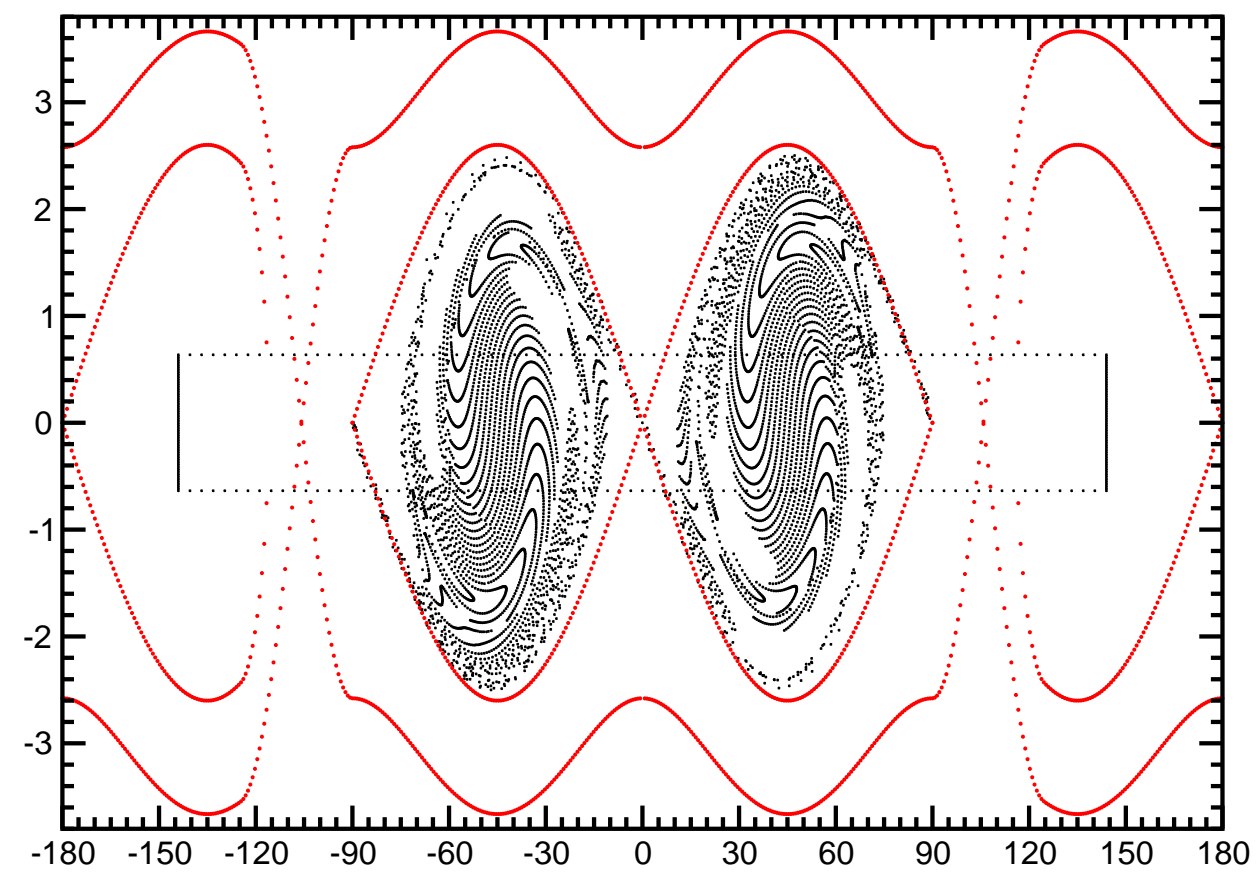

Figure 24: $6 \mathrm{~ms}$ squeeze to half turn followed by $6 \mathrm{~ms}$ harmonic 4 capture. 


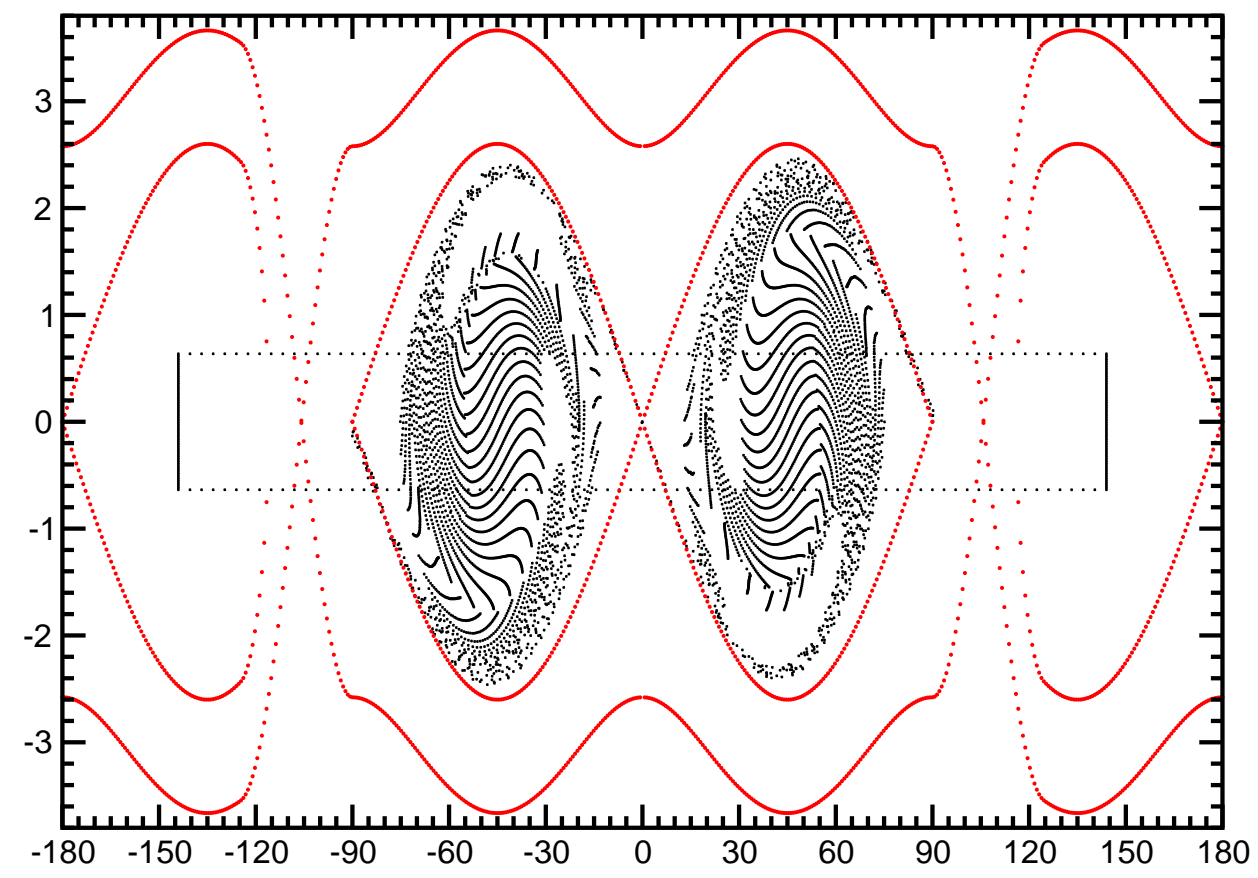

Figure 25: $7 \mathrm{~ms}$ squeeze to half turn followed by $5 \mathrm{~ms}$ harmonic 4 capture. 